\title{
REDUCED DYNAMICS OF STOCHASTICALLY PERTURBED GRADIENT FLOWS*
}

\author{
IBRAHIM FATKULLIN ${ }^{\dagger}$, GREGOR KOVAČIČ ${ }^{\ddagger}$, AND ERIC-VANDEN-EIJNDEN ${ }^{\S}$
}

\author{
Dedicated to the sixtieth birthday of Professor Andrew Majda
}

\begin{abstract}
We consider stochastically perturbed gradient flows in the limit when the amplitude of random fluctuations is small relative to the typical energy scale in the system and the minima of the energy are not isolated but form submanifolds of the phase space. In this case the limiting dynamics may be described in terms of a diffusion process on these manifolds. We derive explicit equations for this limiting dynamics and illustrate them on a few finite-dimensional examples. Finally, we formally extrapolate the reduction technique to several infinite-dimensional examples and derive equations of the stochastic kink motion in Allen-Cahn-type systems.
\end{abstract}

Key words. Stochastic gradient flows, reduced dynamics, stochastic Allen-Cahn, kinks.

AMS subject classifications. 34F05, 60H10, 60H15, 93E03.

\section{Introduction}

The temporal evolution of a number of physical systems can be modeled by stochastically perturbed gradient flows on some prescribed (free) energy landscape. When the amplitude of the random perturbations is small, their influence is in first approximation negligible on the intrinsic time-scale of the deterministic gradient flow. On longer time-scales, however, the situation changes and the random perturbations have a nontrivial effect on the dynamics. A typical situation is when the energy landscape has isolated minima. In this case, the deterministic part of the dynamics drives the system by steepest descent to the vicinity of one of these minima, where it remains for a very long period of time. From time to time, however, the random perturbations push the system significantly up in energy and away from this minimum. If the landscape is non convex, eventually the system manages to escape the basin of attraction of the minimum it is currently in and finds its way towards the location of another minimum. The cycle can then repeat. The typical time of escape from the location of one minimum to another can be estimated from the theory of large deviations [16] and is exponentially long relative to the height of the energy barrier between these minima measured in units of the amplitude of the random perturbation. Additional information, such as the paths of maximum likelihood by which the transitions between minima occur, is also provided by large deviation theory $[16,8]$.

Another interesting class of situations, which are the ones we will focus on in this paper, arises when the energy landscape does not have isolated minima but rather a manifold of minima. In fact, it is enough to assume that the energy function can be decomposed as $E=V+\epsilon U$, where $\epsilon$ is a parameter measuring the amplitude of the random perturbations and $V$ has a manifold of minimizers. In such situations, by rescaling time as $t \mapsto t / \epsilon$ and assuming that $\epsilon$ is small the system is first quickly attracted to the vicinity of this manifold by the deterministic part of the dynamics

\footnotetext{
*Received: December 2, 2008; accepted (in revised version): March 19, 2009.

†Department of Mathematics, 617 N Santa Rita Ave, PO Box 210089, Tucson, AZ 85718 (ibrahim@math.arizona.edu).

${ }^{\ddagger}$ Department of Mathematical Sciences, Rensselaer Polytechnic Institute, Troy, NY 12180 (kovacg@rpi.edu).

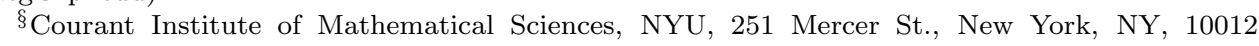
(eve2@cims.nyu.edu).
} 
associated with steepest descent on $V$. Afterwards the system displays a nontrivial dynamics in the neighborhood of the manifold which is governed by the interplay of the action of $U$ and that of the random perturbations. This is the dynamics that we are interested in studying in this paper.

As we will see, in the limit of small $\epsilon$ it is possible to describe the dynamics in terms of the variables parametrizing the manifold of the minimizers of $V$ rather than the entire (often much larger) phase space. This is much in the spirit of singular perturbation theory developed for deterministic dynamical systems by Fenichel [14], where, up to small corrections, the dynamics in the neighborhood of attracting invariant slow manifolds may be reduced to evolution on them (for the general theory regarding stochastic systems see, e.g., [2] and references within). In particular an appropriate "calculus" was developed for the differential equations describing these dynamics. Here we consider the stochastic case and derive stochastic ordinary differential equations (SODE) governing the reduced dynamics. In particular, we extend (the attracting sub-case of) Fenichel's singular-perturbation "calculus" to the stochastic case by building upon a general theorem by Katzenberger [18]. Further on, we also apply these ideas in the infinite-dimensional setting of stochastic partial differential equations (SPDE), although without a rigorous justification.

The present paper has two principal components: first we rigorously derive the reduced equations in the finite-dimensional situation, i.e., when the phase space of the system is finite-dimensional (i.e the evolution are described by an SODE). As a particular example we consider a model illustrating temperature-induced change of equilibrium in a two-dimensional system. Then we extrapolate the finite-dimensional results to an infinite-dimensional setting, i.e., when the dynamics of the entire system is described by a stochastic partial differential equation (SPDE). In this case we do not provide complete proofs and limit ourselves to highlighting the key ideas and methods using the stochastic Allen-Cahn type equations (1+1 space-time dimensions) that describe phase separation dynamics. We demonstrate how in an appropriate limit these SPDEs may be reduced to diffusion-annihilation processes describing the motion of phase separation points.

The remainder of this paper is organized as follows. In section 2 we describe the general setting for our problem and state our main result, Theorem 2.2. In section 3 we illustrate this result via application to examples. In section 4 we formally generalize our result to the infinite-dimensional setting and, as an application, we consider the stochastic Allen-Cahn equation with additive noise. Finally, in section 5 we provide the proofs of Theorem 2.2 and other results.

\section{Main results in finite dimensions}

We will consider $n$-dimensional stochastic differential equations of the form

$$
\mathrm{d} \boldsymbol{X}_{t}=-D \nabla E^{\epsilon}\left(\boldsymbol{X}_{t}\right) \mathrm{d} t+\sqrt{2 \tau} \sigma \mathrm{d} \boldsymbol{W}_{t} .
$$

Here $E^{\epsilon}(\boldsymbol{x})$ is referred to as the energy, $\tau$ as the temperature, and the symmetric positive-definite $n \times n$ matrix $D$ as the diffusivity; $\boldsymbol{W}_{t}$ is a $d$-dimensional Brownian motion (Wiener process), and we assume that the $n \times d$ matrix $\sigma$ is related to diffusivity as $D=\sigma \sigma^{T}$.

The superscript $\epsilon$ in $E^{\epsilon}(\boldsymbol{x})$ indicates that the energy depends on a control parameter $\epsilon$. Specifically, we are interested in situations where the energy can be decomposed as

$$
E^{\epsilon}(\boldsymbol{x})=U(\boldsymbol{x})+\frac{1}{\epsilon} V(\boldsymbol{x}) .
$$


When $\epsilon$ is small, the decomposition (2.2) means that the energy has widely separated scales and $U(\boldsymbol{x})$ and $V(\boldsymbol{x})$ will be referred to as the slow and fast energies, respectively. Intuitively, one would (correctly) expect that the dynamics of equation (2.1) will relax along the paths of steepest descent towards the minima of $V(\boldsymbol{x})$ on the fast $\mathcal{O}(\epsilon)$ time scale. As explained in the introduction, one then needs to distinguish between the following two cases.

If $V(\boldsymbol{x})$ has a number of isolated minima, the system spends most of the time in the vicinity of these minima and only makes occasional transitions from one minimum to another. The transitions occur on the very long time scale $\mathcal{O}\left(e^{C / \epsilon}\right)$, where the constant $C$ is related to the energy barrier between the minima. The dynamics of the transitions can be described by a Markov chain [16].

In contrast, when the minima of $V(\boldsymbol{x})$ are not isolated but form surfaces (manifolds) in $\mathbb{R}^{n}$, it is natural to expect that nontrivial dynamics will take place very close to these manifolds on the $\mathcal{O}(1)$ time scale (i.e., long before possible transitions between these manifold of minima occur). One can also expect that the description of the $\mathcal{O}(1)$-dynamics can be reduced in this case to a description in terms of the coordinates that parametrize these manifolds. In what is to follow, we will focus on this second situation and derive the reduced equation arising in the limit as $\epsilon \rightarrow 0$.

Specifically, let us consider equation (2.1) on the $n$-dimensional Euclidean space $\mathbb{R}^{n}$ with the energy $E^{\epsilon}(\boldsymbol{x})$ as in (2.2). We assume that the slow potential $U(\boldsymbol{x}) \in C^{2}$ and make the following main assumption about the fast potential $V(\boldsymbol{x})$ :

Assumption 2.1. $V(\boldsymbol{x}) \in C^{5}$ and attains its minimal value on an m-dimensional manifold $\mathcal{M} \subset \mathbb{R}^{n}$ which is $C^{2}$ and can be (locally) parametrized by $m$ coordinates via a mapping $\boldsymbol{\psi}(\boldsymbol{z})$, i.e., any patch $\mathcal{U}$ of $\mathcal{M}$ can be represented as

$$
\mathcal{U}=\left\{\boldsymbol{x} \in \mathbb{R}^{n} \mid \boldsymbol{x}=\boldsymbol{\psi}(\boldsymbol{z}), \boldsymbol{z} \in \mathcal{A} \subset \mathbb{R}^{m}\right\},
$$

where $\mathcal{A}$ is a suitable parameter domain. In addition, the Hessian of $V(\boldsymbol{x})$ has exactly $n-m$ positive eigenvalues when $\boldsymbol{x} \in \mathcal{M}$.

Our main result is a set of equations for a "reduced" process $\boldsymbol{Z}_{t}$ on $\mathbb{R}^{m}$ such that $\boldsymbol{\psi}\left(\boldsymbol{Z}_{t}\right)$ approximates the original process, $\boldsymbol{X}_{t}^{\epsilon}$, for small values of $\epsilon$ in the following sense:

TheORem 2.2. Consider a family of initial conditions $\boldsymbol{X}_{0}^{\epsilon}$ converging to some $\boldsymbol{X}_{0} \in$ $\mathcal{M}$ as $\epsilon \rightarrow 0$ and let $\mathcal{U}$ be a compact neighborhood of $\boldsymbol{X}_{0}$ on $\mathcal{M}$ which is parameterized as in (2.3). Then for all $\delta>0$,

$$
\lim _{\epsilon \rightarrow 0} \mathbb{P}\left(\sup _{0 \leq t \leq T}\left|\boldsymbol{X}_{t}^{\epsilon}-\boldsymbol{\psi}\left(\boldsymbol{Z}_{t}\right)\right|>\delta\right)=0
$$

where $T=\inf \left\{t \geq 0 \mid \boldsymbol{\psi}\left(\boldsymbol{Z}_{t}\right) \notin \mathcal{U}\right\}$ and $\boldsymbol{Z}_{t}$ is the process on $\mathbb{R}^{m}$ that obeys the Stratonovich $S D E$

$$
\mathrm{d} \boldsymbol{Z}_{t}=-\hat{D}\left(\boldsymbol{Z}_{t}\right) \nabla F\left(\boldsymbol{Z}_{t}\right) \mathrm{d} t+\sqrt{2 \tau} \hat{\sigma}\left(\boldsymbol{Z}_{t}\right) \circ \mathrm{d} \boldsymbol{W}_{t}
$$

with the initial condition $\boldsymbol{Z}_{0}$ satisfying $\boldsymbol{X}_{0}=\boldsymbol{\psi}\left(\boldsymbol{Z}_{0}\right)$. Here, the reduced energy $F(\boldsymbol{z})$, reduced diffusivity $\hat{D}(\boldsymbol{z})$, and $\hat{\sigma}(\boldsymbol{z})$ are related to the coefficients of equation (2.1) via the formulas

$$
F(\boldsymbol{z})=U(\boldsymbol{\psi}(\boldsymbol{z}))+\frac{\tau}{2} \ln h(\boldsymbol{z})
$$




$$
\begin{gathered}
\hat{D}_{\alpha \beta}^{-1}(\boldsymbol{z})=\sum_{i, j=1}^{n} \frac{\partial \psi_{i}(\boldsymbol{z})}{\partial z_{\alpha}} D_{i j}^{-1} \frac{\partial \psi_{j}(\boldsymbol{z})}{\partial z_{\beta}}, \\
\hat{\sigma}_{r}^{\alpha}(\boldsymbol{z})=\sum_{i, j=1}^{n} \sum_{\beta=1}^{m} \hat{D}_{\alpha \beta}(\boldsymbol{z}) \frac{\partial \psi_{i}(\boldsymbol{z})}{\partial z_{\beta}} D_{i j}^{-1} \sigma_{r}^{j} .
\end{gathered}
$$

The function $h(\boldsymbol{z})$ in equation (2.6a) is the product of the nonzero eigenvalues of the matrix $J^{T} H(\boldsymbol{\psi}(\boldsymbol{z})) J$, where $H(\boldsymbol{x})$ is the Hessian of $V(\boldsymbol{x})$ at $\boldsymbol{x}=\boldsymbol{\psi}(\boldsymbol{z})$ and $J$ is any $n \times n$ matrix such that $J^{T} J=D$.

Observe that the correction to the energy in (2.6a) is proportional to the temperature, $\tau$, i.e., it is a purely stochastic effect and would not have appeared in the deterministic setting. This correction encodes the influence of the shape of the fast potential $V(\boldsymbol{x})$ in the directions orthogonal to the manifold $\mathcal{M}$. The purely deterministic reduction in the absence of noise corresponds to $\tau=0$.

REmark 2.3. Assuming that $\exp \left\{-E^{\epsilon}(\boldsymbol{x}) / \tau\right\}$ is integrable, the process described by equation (2.1) has the unique Gibbs-type invariant probability measure,

$$
\mu(\mathrm{d} \boldsymbol{x})=\frac{1}{\mathcal{Z}} \exp \left\{-E^{\epsilon}(\boldsymbol{x}) / \tau\right\} \mathrm{d} \boldsymbol{x} .
$$

By a direct computation, one can verify that equation (2.5) then preserves the measure

$$
\hat{\mu}(\mathrm{d} \boldsymbol{z})=\exp \{-F(\boldsymbol{z}) / \tau\} \operatorname{det} \hat{D}^{-1 / 2}(\boldsymbol{z}) \mathrm{d} \boldsymbol{z} .
$$

Provided equation (2.5) can be extended to the entire $\mathcal{M}$, the measure (2.8) is an invariant measure for the process $\boldsymbol{Z}_{t}$. In fact, (2.8) is (up to a scaling factor) the $\epsilon \rightarrow 0$ limit of the invariant measure (2.7) for the original equation (2.1), meaning that the latter converges to a measure concentrated on $\mathcal{M}$, which in the $\boldsymbol{z}$-coordinates can be represented as (2.8). A computation demonstrating this statement and making it precise is carried out in section 5.2.

REMARK 2.4. Note that it is sufficient for the matrix $D$ in equation (2.1) to be positive semi-definite rather than strictly positive-definite. This is the so-called hard constraint scenario: the dynamics prescribed by (2.1) is constrained to a hyperplane which is a translation of the range of $D$ by the vector of initial conditions, $\boldsymbol{X}_{0}$. In this case the entire $\mathbb{R}^{n}$ is representable as a direct sum of the kernel of $D$ and its range, whose every translation is a hyperplane invariant under the dynamics prescribed by equation (2.1). We can thus apply Theorem 2.2 within each such hyperplane and then reformulate the result in terms of the full space $\mathbb{R}^{n}$. Formulas (2.5) and (2.6) remain the same, except that $D^{-1}$ should be replaced by a symmetric matrix, $G$, which inverts $D$ in its range (see Lemma 5.1 in the end of section 5.3), and the $\boldsymbol{z}$ variables parametrize $\tilde{\mathcal{M}}$, which is the intersection of $\mathcal{M}$ (the manifold of the minimizers of the fast potential $V(\boldsymbol{x}))$ with the range of $D$ shifted by $\boldsymbol{X}_{0}$. This procedure can be carried out if all initial conditions, $\boldsymbol{X}_{0}^{\epsilon}$, belong to a single hyperplane.

REMARK 2.5. When the diffusivity matrix $D$ is degenerate, as discussed in the previous remark, the natural description of the reduced problem is frequently obtained in terms of the coordinates on the entire manifold $\mathcal{M}$ rather than on its submanifold, $\tilde{\mathcal{M}}$. In this case, the reduced equation (2.5) must still obey the constraint, in other words, the reduced diffusivity, $\hat{D}(\boldsymbol{z})$, in the natural $\boldsymbol{z}$ coordinates on $\mathcal{M}$ must remain 
degenerate to keep the constraint intact. Thus, formula (2.6b) actually produces some matrix $\hat{G}(\boldsymbol{z})$ (rather than an undefined $\hat{D}^{-1}(\boldsymbol{z})$ ) which then has to be inverted on the tangent space of $\tilde{\mathcal{M}}$ at $\boldsymbol{z}$. The symmetric inverse is, in fact, uniquely defined and may be represented as (skipping $\boldsymbol{z}$ in the arguments)

$$
\hat{D}=P\left[P^{T} \hat{G} P+\tau Q^{T} Q\right]^{-1} P^{T},
$$

where $P(\boldsymbol{z})$ is an arbitrary projector on the tangent space of $\tilde{\mathcal{M}}$ at $\boldsymbol{z}, Q(\boldsymbol{z})=I-P(\boldsymbol{z})$, and $\tau>0$. Note that $(2.9)$ reduces to the identity $\hat{D}(\boldsymbol{z})=\hat{G}^{-1}(\boldsymbol{z})$ whenever $P(\boldsymbol{z})=I$, e. g., when the $\boldsymbol{z}$-variables only parametrize $\tilde{\mathcal{M}}$ rather than the entire manifold $\mathcal{M}$. The derivation of formula (2.9) is presented in section 5.3. One of the reasons we present this formula is that this version of the hard constraint scenario is typical in the infinite-dimensional setting and an analog of (2.9) will be used when we discuss the constrained Allen-Cahn equation (section 4.3).

REMARK 2.6. Finally, note that we consider equation (2.1) to be a prototypical example of a larger class of Itô diffusion processes of the form

$$
\mathrm{d} \boldsymbol{X}_{t}=\left[\boldsymbol{v}\left(\boldsymbol{X}_{t}\right)-D\left(\boldsymbol{X}_{t}\right) \nabla E\left(\boldsymbol{X}_{t}\right)+\tau \nabla \cdot D\left(\boldsymbol{X}_{t}\right)\right] \mathrm{d} t+\sqrt{2 \tau} \sigma\left(\boldsymbol{X}_{t}\right) \mathrm{d} \boldsymbol{W}_{t},
$$

where $D\left(\boldsymbol{X}_{t}\right)=\sigma\left(\boldsymbol{X}_{t}\right) \sigma^{T}\left(\boldsymbol{X}_{t}\right)$ and $\nabla \cdot[\boldsymbol{v}(\boldsymbol{x}) \rho(\boldsymbol{x})]=0$, with $\rho(\boldsymbol{x})$ being the density of the invariant measure of the form (2.7). This is the most general class of diffusion processes that preserve the invariant measure $\rho(\boldsymbol{x}) \mathrm{d} \boldsymbol{x}$. Equ. (2.1) is special in that its dynamics obey the detailed balance condition $(\boldsymbol{v}(\boldsymbol{x}) \equiv 0$, i.e., the probability flux through any surface vanishes at equilibrium) and that the noise term in it has $\boldsymbol{x}$-independent correlation functions. Physically, this last statement means that the auto-correlation functions of the stochastic Langevin forces in equation (2.1) are independent of the instantaneous values of the macroscopic variables. Analysis of equation (2.10) and its final result are quite similar to those presented here for equation (2.1), however, the calculation is more cumbersome and is omitted.

The proof of Theorem 2.2 and propositions from the remarks above will be presented in section 5. First, however, we consider a number of finite-dimensional examples that illustrate specific features of the reduction described in Theorem 2.2, as well as its formal extension to several infinite-dimensional examples. In these latter examples, Theorem 2.2 serves as a guiding principle in the calculations, even though the corresponding rigorous theory is as yet missing.

\section{Examples}

In this section, we present three characteristic examples of the theory developed in the previous section. Two of them illustrate the properties of the noise-induced correction to the energy.

3.1. Effective drift and thermally-induced change of equilibrium. Let us consider a two-dimensional example that illustrates the importance of the term $(\tau / 2) \ln h(\boldsymbol{z})$ in the reduced energy. We will see that quite nontrivial and unexpected phenomena may occur due to this term. We use $\boldsymbol{x}=(x, y) \in \mathbb{R}^{2}$ to denote our degrees of freedom.

Consider the following slow and fast potentials:

$$
U(x, y)=\frac{x^{2}}{2}, \quad V(x, y)=\frac{1}{2} \frac{y^{2}}{1+x^{2}},
$$




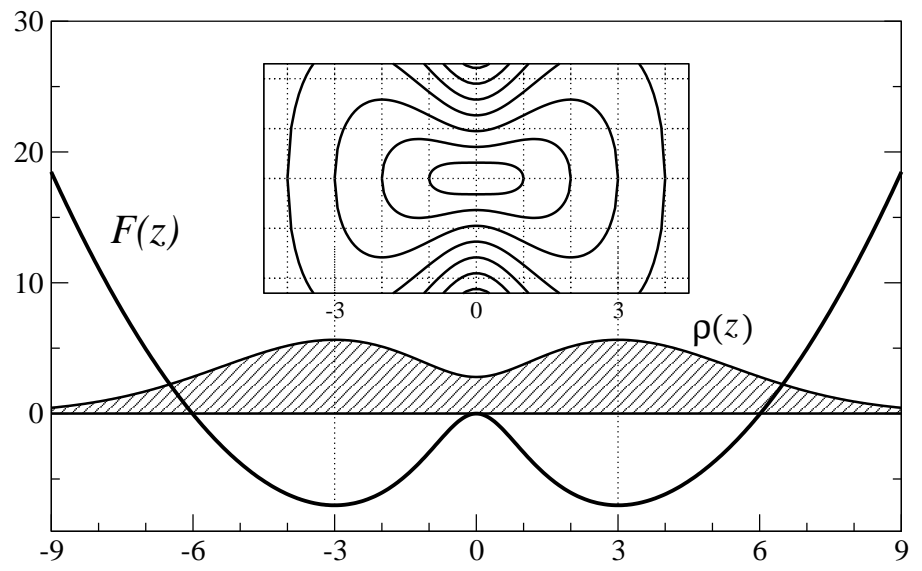

FIG. 3.1. The main plot displays the effective potential $F(z)$ and the invariant measure for the reduced process, $\rho(x)=\mathrm{e}^{-F(z) / \tau} / Z$ (stretched for the purposes of illustration). The subplot displays the level sets of the true potential $E(x, y)=U(x, y)+V(x, y) / \epsilon$.

and let $\sigma$ and $D$ be the $2 \times 2$ identity matrices. In this case, the dynamics is given explicitly by the equations

$$
\left\{\begin{array}{l}
\mathrm{d} X_{t}=-X_{t} \mathrm{~d} t+\frac{1}{\epsilon} X_{t} Y_{t}^{2}\left(1+X_{t}^{2}\right)^{-2} \mathrm{~d} t+\sqrt{2 \tau} \mathrm{d} W_{t}^{(1)}, \\
\mathrm{d} Y_{t}=-\frac{1}{\epsilon} Y_{t}\left(1+X_{t}^{2}\right)^{-1} \mathrm{~d} t+\sqrt{2 \tau} \mathrm{d} W_{t}^{(2)} .
\end{array}\right.
$$

The slow manifold $\mathcal{M}$ here is the $x$-axis, so we naturally parametrize it by the $x$ coordinate, setting $(x, y)=\boldsymbol{\psi}(z)=(z, 0)$. (The $z$-variable is nevertheless used to avoid any confusion between the original and the reduced equations.) Computing the quantities prescribed in formulas (2.6), we find $\hat{D}(z) \equiv 1, \hat{\sigma}(z) \equiv(1,0)$, and

$$
F(z)=\frac{z^{2}}{2}-\frac{\tau}{2} \ln \left(1+z^{2}\right) .
$$

Thus, the reduced dynamics obey the equation

$$
\mathrm{d} Z_{t}=-Z_{t} \mathrm{~d} t+\frac{\tau Z_{t}}{1+Z_{t}^{2}} \mathrm{~d} t+\sqrt{2 \tau} \mathrm{d} W_{t}^{(1)} .
$$

We now proceed to analyze the structure of the reduced (or effective) energy (3.3) a bit closer. For $\tau \leq 1,(3.3)$ has a unique minimum at $z=0$. This minimum undergoes a saddle-node bifurcation at $\tau=1$ and becomes a local maximum, while two minima at $z= \pm \sqrt{\tau-1}$ emerge. The gradient flow dynamics (3.4) therefore have qualitatively different features depending on whether $\tau \leq 1$ or $\tau>1$ : in the first case, the drift is towards the origin, while in the second case, the drift is away from the origin and towards the minima of the effective energy. This is what may be called the entropic neck effect. Even though the actual energy $E(\boldsymbol{x})=U(\boldsymbol{x})+V(\boldsymbol{x}) / \epsilon$ has a unique minimum at $\boldsymbol{x}=0$, for small $\epsilon$ and large $\tau$, the system prefers to be situated near the minima $x= \pm \sqrt{\tau-1}$ of the effective energy, $F(z)$ (recall $z \equiv x$ ); see figure 3.1 for the illustration. The reason for this is that the "width" of the phase space in $y$ direction (the number of compatible micro-configurations in the language of statistical physics) is much smaller near $x=0$ than near $x= \pm \sqrt{\tau-1}$. Thus, effectively, when $\tau>1$, the system is less likely to be found near $x=0$. 
3.2. Brownian motion on a manifold. This is a version of the (last) example from [18]. Let $\mathcal{M}$ be a smooth $m$-dimensional submanifold of $\mathbb{R}^{n}$ (locally) parametrized by a function $\boldsymbol{\psi}: \mathbb{R}^{m} \rightarrow \mathbb{R}^{n}$,

$$
U(\boldsymbol{x})=0, \quad V(\boldsymbol{x})=\frac{1}{2}[\operatorname{dist}(\boldsymbol{x}, \mathcal{M})]^{2}
$$

where $\operatorname{dist}(\cdot, \cdot)$ denotes the usual Euclidean distance. Let $D$ and $\sigma$ be the $n \times n$ identity matrices. (Note that while $V(\boldsymbol{x})$ is not necessarily smooth in the entire $\mathbb{R}^{n}$, it is smooth in a sufficiently small neighborhood of $\mathcal{M}$.) From formulas (2.6) we obtain that the reduced energy $F(\boldsymbol{z})=0$,

$$
\hat{\sigma}_{r}^{\alpha}(\boldsymbol{z})=\sum_{\beta=1}^{m} \hat{D}_{\alpha \beta}(\boldsymbol{z}) \frac{\partial \psi_{r}(\boldsymbol{z})}{\partial z_{\beta}}, \quad \hat{D}_{\alpha \beta}^{-1}(\boldsymbol{z})=\sum_{i=1}^{n} \frac{\partial \psi_{i}(\boldsymbol{z})}{\partial z_{\alpha}} \frac{\partial \psi_{i}(\boldsymbol{z})}{\partial z_{\beta}} .
$$

Therefore, the equation for the reduced process $\boldsymbol{Z}_{t}$ can be written as

$$
\mathrm{d} Z_{t}^{\alpha}=\sqrt{2 \tau} \sum_{r=1}^{n} \sum_{\beta=1}^{m} \hat{D}_{\alpha \beta}(\boldsymbol{z}) \frac{\partial \psi_{r}(\boldsymbol{z})}{\partial z_{\beta}} \circ \mathrm{d} W_{t}^{r} .
$$

A straightforward computation shows that the generator of this process is the Laplace-Beltrami operator on $\mathcal{M}$ (up to the factor of $\tau$ ), on which $\hat{D}_{\alpha \beta}^{-1}(\boldsymbol{z})$ plays the role of a metric tensor, i.e., $\mathcal{M}$ inherits its metric from $\mathbb{R}^{n}$. This implies that $\boldsymbol{Z}_{t}$ is a Brownian motion on $\mathcal{M}$ (modulo the scaling factor of $\sqrt{2 \tau}$ ). This fact, however, is easier to see if we write equation (3.7) for $\boldsymbol{Z}_{t}$ in terms of the original $\boldsymbol{x}$-coordinates in $\mathbb{R}^{n}$ (see equation (5.18) below). This is because we can use the wellknown result that Brownian motion on a submanifold $\mathcal{M}$ of $\mathbb{R}^{n}$ satisfies the equation $\mathrm{d} \boldsymbol{X}_{t}=P\left(\boldsymbol{X}_{t}\right) \circ \mathrm{d} \boldsymbol{W}_{t}$, where $P(\boldsymbol{x})$ is the orthogonal projector on the tangent space of $\mathcal{M}$ at $\boldsymbol{x}$ [23]. For the process $\boldsymbol{X}_{t}=\boldsymbol{\psi}\left(\boldsymbol{Z}_{t}\right)$, we have

$$
\mathrm{d} \boldsymbol{X}_{t}=\mathrm{d} \boldsymbol{\psi}\left(\boldsymbol{Z}_{t}\right)=\sqrt{2 \tau} P\left(\boldsymbol{Z}_{t}\right) \circ \mathrm{d} \boldsymbol{W}_{t},
$$

where $P$ is the matrix with entries

$$
P_{i j}(\boldsymbol{z})=\sum_{\alpha, \beta=1}^{m} \frac{\partial \psi_{i}(\boldsymbol{z})}{\partial z_{\alpha}} \hat{D}_{\alpha \beta}(\boldsymbol{z}) \frac{\partial \psi_{j}(\boldsymbol{z})}{\partial z_{\beta}} .
$$

Observing that $P(\boldsymbol{z})$ is exactly the required orthogonal projector, we immediately verify the assertion.

3.3. Soft constraint to a manifold. Now consider the following modification of the previous example. Let the manifold $\mathcal{M}$ be given as the zero level set (which is assumed to be non-degenerate) of some smooth function $\boldsymbol{\theta}: \mathbb{R}^{n} \rightarrow \mathbb{R}^{n-m}$,

$$
U(\boldsymbol{x})=0, \quad V(\boldsymbol{x})=\frac{1}{2}|\boldsymbol{\theta}(\boldsymbol{x})|^{2} .
$$

Let $D$ and $\sigma$, again, be the $n \times n$ identity matrices. As in the previous example, we obtain that the reduced diffusivity and the matrix $\sigma_{r}^{\alpha}(\boldsymbol{z})$ are given by formulas (3.6), however, there now exists a nontrivial effective energy contribution

$$
F(\boldsymbol{z})=\frac{\tau}{2} \ln h(\boldsymbol{z})
$$


where the function $h(\boldsymbol{z})$ is the product of nonzero eigenvalues of the Hessian of $V(\boldsymbol{x})$ evaluated at $\boldsymbol{x}=\boldsymbol{\psi}(\boldsymbol{z})$. Observe that $h(\boldsymbol{z})$ may be computed explicitly as the sum of rank $n-m$ principal minors of the Hessian,

$$
H_{i j}(\boldsymbol{x})=\sum_{p=1}^{n-m}\left[\theta^{p}(\boldsymbol{x}) \frac{\partial^{2} \theta^{p}(\boldsymbol{x})}{\partial x^{i} \partial x^{j}}+\frac{\partial \theta^{p}(\boldsymbol{x})}{\partial x^{i}} \frac{\partial \theta^{p}(\boldsymbol{x})}{\partial x^{j}}\right]_{\boldsymbol{x}=\boldsymbol{\psi}(\boldsymbol{z})} .
$$

In particular, if $m=n-1$, i.e., if $\theta(\boldsymbol{x})$ is a scalar function, $h(\boldsymbol{z})=\operatorname{Tr} H(\boldsymbol{\psi}(\boldsymbol{z}))$. Summarizing all of the above we see that the reduced process $\boldsymbol{Z}_{t}$ satisfies

$$
\mathrm{d} Z_{t}^{\alpha}=-\sum_{\beta=0}^{m} \hat{D}_{\alpha \beta}\left(\boldsymbol{Z}_{t}\right) \frac{\partial F\left(\boldsymbol{Z}_{t}\right)}{\partial z^{\beta}} \mathrm{d} t+\sqrt{2 \tau} \sum_{r=1}^{n} \sum_{\beta=1}^{m} \hat{D}_{\alpha \beta}\left(\boldsymbol{Z}_{t}\right) \frac{\partial \psi_{r}\left(\boldsymbol{Z}_{t}\right)}{\partial z_{\beta}} \circ \mathrm{d} W_{t}^{r} .
$$

Note the difference from equation (3.7): there is now a drift term due to the nontrivial contribution from the Hessian of the fast potential.

From the computational point of view sometimes it is convenient to remain in the original coordinates of $\mathbb{R}^{n}$, in which case for the process $\boldsymbol{X}_{t}=\boldsymbol{\psi}\left(\boldsymbol{Z}_{t}\right)$ we have

$$
\mathrm{d} \boldsymbol{X}_{t}=-P\left(\boldsymbol{X}_{t}\right) \nabla \hat{F}\left(\boldsymbol{X}_{t}\right) \mathrm{d} t+\sqrt{2 \tau} P\left(\boldsymbol{X}_{t}\right) \circ \mathrm{d} \boldsymbol{W}_{t} .
$$

Here $\hat{F}(\boldsymbol{x})$ is the same reduced energy expressed in $\boldsymbol{x}$-coordinates, i.e., $\hat{F}(\boldsymbol{\psi}(\boldsymbol{z}))=$ $F(\boldsymbol{z})$, and the gradient is taken with respect to the $\boldsymbol{x}$-variables. Note that the projector $P(\boldsymbol{x})$ may be computed without referring to the map $\boldsymbol{\psi}(\boldsymbol{z})$, using the the prescribed map $\boldsymbol{\theta}(\boldsymbol{x})$ alone:

$$
P_{i j}(\boldsymbol{x})=\delta_{i j}-\sum_{p, q=1}^{n-m} \frac{\partial \theta^{p}(\boldsymbol{x})}{\partial x^{i}}\left[T^{-1}(\boldsymbol{x})\right]_{p q} \frac{\partial \theta^{q}(\boldsymbol{x})}{\partial x^{j}},
$$

where the matrix $T(\boldsymbol{x})$ is given by

$$
T_{p q}(\boldsymbol{x})=\sum_{i=1}^{n} \frac{\partial \theta^{p}(\boldsymbol{x})}{\partial x^{i}} \frac{\partial \theta^{q}(\boldsymbol{x})}{\partial x^{i}}
$$

Finally, note that if the slow potential $U(\boldsymbol{x})$ is nonzero, it is added to the reduced energy $\hat{F}(\boldsymbol{x})$ in (3.14) while the structure of the equation remains unaffected (similarly, $U(\boldsymbol{\psi}(\boldsymbol{z}))$ is then added to $F(\boldsymbol{z})$ in $(3.13))$.

\section{Infinite-dimensional generalizations}

Motivated by the finite-dimensional (SODE) results, we now heuristically apply the same ideas to infinite-dimensional (SPDE) problems. This approach has been adopted, e. g., in [17], where a rigorous treatment of the so-called single-kink solution of the Allen-Cahn equation (see below) has been carried out. Additional technical details regarding the interpretation of the SPDEs that we consider here, existence and uniqueness of solutions, etc., may be found in [20, 12, 22, 19].

4.1. Stochastic Allen-Cahn equation. Consider the following stochastic nonlinear heat equation:

$$
u_{t}(x, t)=u_{x x}+\frac{1}{\epsilon^{2}}\left(u-u^{3}\right)+\sqrt{2 \tau} \xi(x, t)
$$


for a real-valued function $u(x, t)$ whose absolute value tends to 1 as $x \rightarrow \pm \infty, t>0$. Here $\epsilon$ and $\tau$ are parameters, $\xi(x, t)$ is a spatio-temporal white noise (it has to be truncated when $|x|$ is sufficiently large to have (4.1) well-posed). Alternatively, (4.1) may be considered on a finite interval $[0, L]$, e.g., with periodic boundary conditions. This issue of boundary conditions is technical and does not affect the phenomena that we study here, thus for the sake of clarity we first consider (4.1) on the whole line and switch to the finite interval in the subsequent examples.

The similarity of (4.1) to our archetypal SDE (2.1) becomes apparent once we observe that (4.1) may be represented as

$$
u_{t}(x, t)=-\frac{\delta \mathcal{E}^{\epsilon}[u]}{\delta u(x)}+\sqrt{2 \tau} \xi(x, t)
$$

where $\delta / \delta u(x)$ denotes the variational (Euler-Lagrange) derivative, and $\mathcal{E}^{\epsilon}[u]$ is the Ginzburg-Landau energy,

$$
\mathcal{E}^{\epsilon}[u]=\int_{\mathbb{R}}\left[\frac{1}{2} u_{x}^{2}+\frac{1}{4 \epsilon^{2}}\left(1-u^{2}\right)^{2}\right] \mathrm{d} x .
$$

Thus equation (4.1) has a gradient flow structure, just like the finite-dimensional equation (2.1). Rescaling the variables by setting $\tilde{x}=x / \epsilon, \tilde{t}=t / \epsilon$, and $\tilde{u}(\tilde{x}, \tilde{t})=u(x, t)$, we may rewrite the equation (4.1) as

$$
\tilde{u}_{\tilde{t}}(\tilde{x}, \tilde{t})=\frac{1}{\epsilon}\left(\tilde{u}_{\tilde{x} \tilde{x}}+\tilde{u}-\tilde{u}^{3}\right)+\sqrt{2 \tau} \tilde{\xi}(\tilde{x}, \tilde{t}),
$$

where $\tilde{\xi}(\tilde{x}, \tilde{t})$ is a different white noise. In this form (4.1) is formally analogous to a finite-dimensional system where the whole energy is fast and is given by

$$
\tilde{\mathcal{E}}^{\epsilon}[\tilde{u}]=\frac{1}{\epsilon} \mathcal{V}[\tilde{u}], \quad \mathcal{V}[\tilde{u}]=\int_{\mathbb{R}}\left[\frac{1}{2} \tilde{u}_{\tilde{x}}^{2}+\frac{1}{4}\left(1-\tilde{u}^{2}\right)^{2}\right] \mathrm{d} \tilde{x} .
$$

It is not hard to see that this energy has a continuous set of minimizers, the so-called single-kink functions given by the translates of

$$
v(\tilde{x})= \pm \tanh (\tilde{x} / \sqrt{2}) .
$$

To verify this, we compute their energy, $\mathcal{V}[v]=2 \sqrt{2} / 3$, and observe that in the class of functions tending to \pm 1 as $\tilde{x} \rightarrow \pm \infty$, we have the following lower bound:

$$
\mathcal{V}[\tilde{u}] \geq \frac{1}{\sqrt{2}} \int_{\mathbb{R}}\left(1-\tilde{u}^{2}\right) \tilde{u}_{\tilde{x}} \mathrm{~d} \tilde{x}=\frac{1}{\sqrt{2}} \int_{-1}^{+1}\left(1-\tilde{u}^{2}\right) \mathrm{d} \tilde{u}=\frac{2 \sqrt{2}}{3} .
$$

Since the energy is constant on the set of single-kink functions and the deviations are $\mathcal{O}(1 / \epsilon)$ expensive in the energy (see section 5.4 for a precise energy bound of this type), this set is the analog of the manifold $\mathcal{M}$, and the small- $\epsilon$ dynamics reduces to the noise-induced motion of the kink.

Parametrizing the single-kink functions by the kink location, $\tilde{Z}$ (we use tilde to indicate that the kink coordinate is prescribed in the rescaled space), we now compute the analogues of the ingredients from (2.5). Instead of the matrix $D$ here we have the identity operator, so we compute $\hat{D}^{-1}(\tilde{z})$ (just a number for the single kink-functions) as in $(2.6 \mathrm{~b})$, except the summation now transforms into integration:

$$
\hat{D}^{-1}(\tilde{z})=\int_{\mathbb{R}}\left[\frac{\partial v(\tilde{x}-\tilde{z})}{\partial \tilde{z}}\right]^{2} \mathrm{~d} \tilde{x}=\frac{2 \sqrt{2}}{3} .
$$




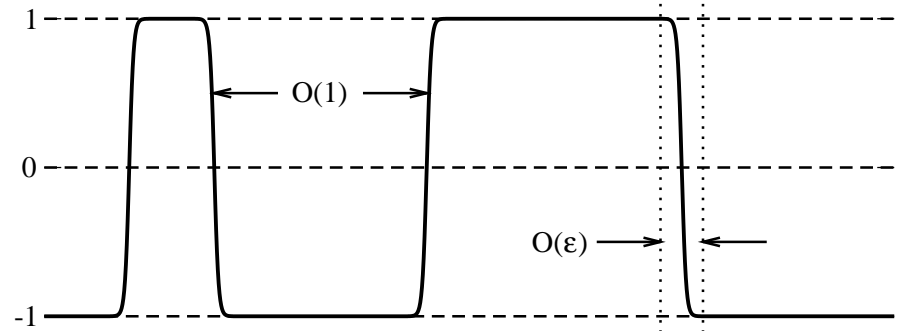

FIG. 4.1. A typical multi-kink function, $v(\tilde{x} \mid \tilde{\boldsymbol{Z}})$, given by the equation (4.12) depicted in the original scaling $x=\epsilon \tilde{x}$. The distance between the kinks is of $\mathcal{O}(1)$, while the width of each transition layer is of $\mathcal{O}(\epsilon)$.

Similarly, we can compute the reduced "matrix" $\hat{\sigma}(\tilde{z})$. It is, however, convenient to immediately contract $\hat{\sigma}(\tilde{z})$ with the noise obtaining a process $\dot{\hat{\sigma}}_{\tilde{t}}(\tilde{z})-$ an equivalent of $\hat{\sigma}(\boldsymbol{z}) \dot{W}_{t}$ from the finite-dimensional case:

$$
\dot{\hat{\sigma}}_{\tilde{t}}(\tilde{z})=\frac{3}{2 \sqrt{2}} \int_{\mathbb{R}} \frac{\partial v(\tilde{x}-\tilde{z})}{\partial \tilde{z}} \tilde{\xi}(\tilde{x}, \tilde{t}) \mathrm{d} \tilde{x} .
$$

(The integral is understood in the sense of distributions and $\tilde{z}$ plays the role of a parameter.) The analogue of the function $h(\boldsymbol{z})$ here would be the "product of nonzero eigenvalues" of the second variation of $\mathcal{V}[\tilde{u}]$, a Sturm-Liouville operator for which such a quantity is not well-defined (technically, such a quantity may be computed relative to some fixed operator, e.g., $-\partial_{\tilde{x} \tilde{x}}$, which amounts to renormalization via subtracting of an "infinite constant" which does not contribute into the gradient flow dynamics). However the spectrum of this operator does not depend on the kink location, so $h^{\prime}(z)=0$, i.e., $h(z)$ does not contribute into the dynamics.

Combining all these facts, we find that the kink dynamics obeys the following (formally Stratonovich) stochastic differential equation:

$$
\dot{\tilde{Z}}_{\tilde{t}}=\sqrt{2 \tau} \dot{\hat{\sigma}}_{\tilde{t}}\left(\tilde{Z}_{t}\right)=\frac{3 \sqrt{\tau}}{2} \int_{\mathbb{R}} \frac{\partial v\left(\tilde{x}-\tilde{Z}_{\tilde{t}}\right)}{\partial \tilde{z}} \circ \tilde{\xi}(\tilde{x}, \tilde{t}) \mathrm{d} \tilde{x} .
$$

Now observe that this equation may also be understood in the Itô sense: the Stratonovich correction vanishes. Therefore we may replace the Gaussian process $\hat{\sigma}_{\tilde{t}}(\tilde{z})$ by a simple Brownian motion with the matching covariance. Thus we obtain that the kink motion is equivalent in law to the solution of

$$
\mathrm{d} \tilde{Z}_{\tilde{t}}=\left[\frac{3 \tau}{\sqrt{2}}\right]^{1 / 2} \mathrm{~d} \tilde{W}_{\tilde{t}}, \quad \text { or } \quad \mathrm{d} Z_{t}=\left[\frac{3 \epsilon \tau}{\sqrt{2}}\right]^{1 / 2} \mathrm{~d} W_{t}
$$

in the original space-time variables, i.e., for $Z=\epsilon \tilde{Z}$. Rescaling the time yet again, we get (up to a change of the Wiener process) $\mathrm{d} Z_{t^{\prime}}=\mathrm{d} W_{t^{\prime}}$ for $t^{\prime}=3 \epsilon \tau t / \sqrt{2}$.

More generally, we can construct the so-called multi-kink functions

$$
\tilde{u}(\tilde{x}) \approx v(\tilde{x} \mid \tilde{\boldsymbol{Z}})=\prod_{\alpha=1}^{N} v\left(\tilde{x}-\tilde{Z}_{\alpha}\right),
$$

where $\tilde{\boldsymbol{Z}}=\left(\tilde{Z}_{1}, \ldots, \tilde{Z}_{N}\right)$ is the vector of kink locations (again, the tilde indicates that these locations are prescribed in the rescaled space), and " $\approx$ " denotes equality up to 
the terms of $\mathcal{O}\left(\exp \left\{-\Delta_{\min }\right\}\right)$ - exponentially small relative to the minimal distance between some two successive kinks. In the neighborhood of such $N$-kink functions the energy (4.5) may be represented as

$$
\mathcal{V}[v(\tilde{x} \mid \tilde{\boldsymbol{Z}})]=\frac{2 \sqrt{2}}{3} N+\mathcal{O}\left(\exp \left\{-\sqrt{2} \Delta_{\min }\right\}\right) .
$$

Now observe that provided the kinks are located on the $\mathcal{O}(1)$ distances in the original function $u(x)$, the distance between the kinks in $\tilde{u}(\tilde{x})$ is of $\mathcal{O}(1 / \epsilon)$, and thus the second term in (4.13) is vanishingly small and may be neglected on the time scales polynomially slow in $\epsilon$ (see, e.g., $[6,5,9]$ for details regarding the deterministic kink motion). Further on, the first term in (4.13) is independent of the kink locations, $\tilde{Z}_{\alpha}$, while expression (4.5) indicates that the deviations from this neighborhood are $\mathcal{O}(1 / \epsilon)$ expensive in the energy. Thus we see that the situation is again formally identical to the finite-dimensional case. As before, we parametrize the set of multi-kink solutions by the kink locations $\tilde{\boldsymbol{Z}}$ for which we compute the analogues of the ingredients from (2.5). For the reduced matrix $\hat{D}_{\alpha \beta}^{-1}(\tilde{\boldsymbol{z}})$ we obtain

$$
\hat{D}_{\alpha \beta}^{-1}(\tilde{\boldsymbol{z}})=\int_{\mathbb{R}} \frac{\partial v(\tilde{x} \mid \tilde{\boldsymbol{z}})}{\partial \tilde{z}_{\alpha}} \frac{\partial v(\tilde{x} \mid \tilde{\boldsymbol{z}})}{\partial \tilde{z}_{\beta}} \mathrm{d} \tilde{x}=\frac{2 \sqrt{2}}{3} \delta_{\alpha \beta}+\mathcal{O}\left(\exp \left\{-\sqrt{2} \Delta_{\min }\right\}\right) .
$$

As in the single-kink situation, while $\Delta_{\min }=\mathcal{O}(1 / \epsilon)$, the function $h(\boldsymbol{z})$ is constant in $\boldsymbol{z}$ and therefore it does not contribute to dynamics: the spectral problem for the second variation of the energy is essentially a superposition of independent translationinvariant problems for separate kinks. Finally we compute the stochastic processes driving the kink dynamics:

$$
\dot{\hat{\sigma}}_{\tilde{t}}^{\alpha}(\tilde{\boldsymbol{z}}) \approx \frac{3}{2 \sqrt{2}} \int_{\mathbb{R}} \frac{\partial v(\tilde{x} \mid \tilde{\boldsymbol{z}})}{\partial \tilde{z}_{\alpha}} \xi(\tilde{x}, \tilde{t}) \mathrm{d} \tilde{x} .
$$

Again, we verify that equations of the kink motion may be understood in the Itô sense and obtain the analogue of (4.11), immediately in the original scaling:

$$
\mathrm{d} \boldsymbol{Z}_{t}=\left[\frac{3 \epsilon \tau}{\sqrt{2}}\right]^{1 / 2} \mathrm{~d} \boldsymbol{W}_{t} .
$$

Setting $t^{\prime}=3 \epsilon \tau t / \sqrt{2}$, we obtain $\mathrm{d} \boldsymbol{Z}_{t^{\prime}}=\mathrm{d} \boldsymbol{W}_{t^{\prime}}$. These equations are valid until the distance between some of the kinks becomes of $\mathcal{O}(\epsilon)$. This process leads to the mutual annihilation of two kinks (which is not considered here), while the remaining kinks keep moving according to (4.16). Let us also mention that the formal asymptotic analysis with the derivation of the stochastic kink dynamics has been carried out in $[25]$.

4.2. Allen-Cahn equation with drift. Now consider a slight modification of the stochastic Allen-Cahn equation,

$$
u_{t}(x, t)=u_{x x}+\frac{1}{\epsilon^{2}}(u-\epsilon c / \sqrt{2})\left(1-u^{2}\right)+\sqrt{2 \tau} \xi(x, t),
$$

where $c$ is a parameter (the standard Allen-Cahn equation (4.1) is obtained by setting $c=0)$. The distinguishing feature of equation (4.17) is that the kinks are no longer stationary, or moving exponentially slowly, but have a finite velocity $c$. This can be 
seen in a straightforward manner from the fact that the deterministic $(\tau=0)$ equation (4.17) admits the traveling-wave solutions $u(x, t)= \pm \tanh [(x \mp c t) / \epsilon \sqrt{2}]$. In the rescaled variables (same as in the previous section) we have

$$
\tilde{u}_{\tilde{t}}(\tilde{x}, \tilde{t})=\frac{1}{\epsilon}\left(\tilde{u}_{\tilde{x} \tilde{x}}+\tilde{u}-\tilde{u}^{3}\right)-\frac{c}{\sqrt{2}}\left(1-\tilde{u}^{2}\right)+\sqrt{2 \tau} \tilde{\xi}(\tilde{x}, \tilde{t}) .
$$

Thus the deterministic part of equation (4.18) describes a stochastically-perturbed gradient flow generated by the modified Ginzburg-Landau energy,

$$
\tilde{\mathcal{E}}^{\epsilon, c}[\tilde{u}]=\mathcal{U}[\tilde{u}]+\frac{1}{\epsilon} \mathcal{V}[\tilde{u}], \quad \mathcal{U}[\tilde{u}]=\frac{c}{\sqrt{2}} \int\left(\tilde{u}-\tilde{u}^{3} / 3\right) \mathrm{d} \tilde{x} .
$$

Observe that now there exists a nontrivial slow energy contribution, $\mathcal{U}[\tilde{u}]$. (We should point out that, as stated, $\mathcal{U}[\tilde{u}]$ cannot be defined on the whole line: the integral is infinite. This issue may be avoided by either considering the problem on the finite interval or redefining $\mathcal{U}[\tilde{u}]$ by subtracting the energy of some fixed function with the same limit as $\tilde{u}(\tilde{x})$ as $\tilde{x} \rightarrow \pm \infty$.) Computing $\mathcal{U}[\tilde{u}]$ on the multi-kink functions (assuming that the first kink corresponds to the transition from -1 to +1 and removing an irrelevant constant) we obtain

$$
\mathcal{U}[v(\tilde{x} \mid \tilde{\boldsymbol{Z}})] \approx \frac{c 2 \sqrt{2}}{3} \sum_{\alpha=1}^{N}(-1)^{\alpha} \tilde{Z}_{\alpha}
$$

The reduction procedure is carried out identically to the regular Allen-Cahn case, except now there exists a nontrivial slow energy contribution due to (4.20), so instead of (4.16) we find

$$
\mathrm{d} Z_{t}^{\alpha}=(-1)^{\alpha+1} c \mathrm{~d} t+\left[\frac{3 \epsilon \tau}{\sqrt{2}}\right]^{1 / 2} \mathrm{~d} W_{t}^{\alpha} .
$$

Note that in the $\epsilon \rightarrow 0$ limit both the ballistic and the stochastic terms survive only if $c=\mathcal{O}(\epsilon)$. Then, if $c=3 \epsilon \tau c^{\prime} / \sqrt{2}, t^{\prime}=3 \epsilon \tau t / \sqrt{2}$ (same as for the regular Allen-Cahn equation), we obtain $\mathrm{d} Z_{t^{\prime}}^{\alpha}=(-1)^{\alpha+1} c^{\prime} \mathrm{d} t+\mathrm{d} W_{t^{\prime}}^{\alpha}$.

4.3. Constrained Allen-Cahn equation. Let us now consider yet another modification of the equation (4.1):

$$
u_{t}(x, t)=\mathcal{D}\left[u_{x x}+\frac{1}{\epsilon^{2}}\left(u-u^{3}\right)\right]+\sqrt{2 \tau} \mathcal{D} \xi(x, t),
$$

where $u(x)$ is periodic over $x \in[0, L]$, and $\mathcal{D}$ is the projector $\left(\mathcal{D}^{2}=\mathcal{D}\right)$ defined by

$$
\mathcal{D} u(x)=u(x)-\frac{1}{L} \int_{0}^{L} u(x) \mathrm{d} x .
$$

Equ. (4.22) is then called the constrained Allen-Cahn equation, since the presence of the operator $\mathcal{D}$ has the effect that the total integral of the field $u(x, t)$ remains constant (constrained) during evolution. Notice that we now consider a periodic rather than infinite spatial domain - this is needed to have $\mathcal{D}$ well-defined. This requires a few minor adjustments of the arguments involving the multi-kink solutions (e.g., the kinks must come in pairs, the hyperbolic tangent shape is no longer the exact minimizer, 
etc.) however, they are of technical nature and do not affect the results, so we omit them in what follows.

The general motivation for the reduction procedure remains the same as before, except, since $\mathcal{D}$ is degenerate, we proceed along the lines outlined in Remark 2.5, i.e., formally employ the formulas derived for the finite-dimensional hard-constraint scenario. Since $\mathcal{D}$ is a projector, it acts as the identity operator in its range, in particular, it acts as its own inverse. Thus we may compute the matrix $\hat{G}(\boldsymbol{z})$ as

$$
\hat{G}_{\alpha \beta}(\tilde{\boldsymbol{z}})=\int_{0}^{L} \frac{\partial v(\tilde{x} \mid \tilde{\boldsymbol{z}})}{\partial \tilde{z}_{\alpha}} \mathcal{D} \frac{\partial v(\tilde{x} \mid \tilde{\boldsymbol{z}})}{\partial \tilde{z}_{\beta}} \mathrm{d} \tilde{x} \approx \frac{2 \sqrt{2}}{3} \delta_{\alpha \beta}-\frac{2 \epsilon}{L}(-1)^{\alpha+\beta} .
$$

Now we use formula $(2.9)$ to invert $\hat{G}$ in the subspace tangent to the slow manifold, i.e., the multi-kink functions with a given total integral. Since in the $\tilde{\boldsymbol{z}}$-variables the total integral of a multi-kink function can be expressed as

$$
\int_{0}^{L} v(\tilde{x} \mid \tilde{\boldsymbol{Z}}) \mathrm{d} \tilde{x} \approx 2 \sum_{\alpha=1}^{N}(-1)^{\alpha} \tilde{Z}_{\alpha}-\frac{L}{\epsilon}
$$

a projector, $P$, on the tangent spaces of the slow manifold may be written as

$$
P_{\alpha \beta}=\delta_{\alpha \beta}-\frac{1}{N}(-1)^{\alpha+\beta} \text {. }
$$

We can now immediately compute, using formula (2.9), the expressions

$$
P^{T} \hat{G} P=\frac{2 \sqrt{2}}{3} P, \quad \hat{D}=\frac{3 \sqrt{2}}{4} P .
$$

Thus, by the same arguments as for the unconstrained Allen-Cahn equation, we arrive at the following equation describing the motion of the kinks (in the original space-time variables):

$$
\mathrm{d} \boldsymbol{Z}_{t}=\left[\frac{3 \epsilon \tau}{\sqrt{2}}\right]^{1 / 2} P \mathrm{~d} \boldsymbol{W}_{t}
$$

with $P$ given by equation (4.26). On the slow time scale, $t^{\prime}=3 \epsilon \tau t / \sqrt{2}$ (same as for the regular Allen-Cahn equation) and we obtain $\mathrm{d} \boldsymbol{Z}_{t^{\prime}}=P \mathrm{~d} \boldsymbol{W}_{t^{\prime}}$.

Analysis of the constrained Allen-Cahn equation (4.22) is a step towards understanding the kink dynamics of the stochastic Cahn-Hilliard equation [3, 4]:

$$
u_{t}(x, t)=-\partial_{x x}\left[u_{x x}+\frac{1}{\epsilon^{2}}\left(u-u^{3}\right)\right]+\sqrt{2 \tau} \partial_{x} \xi(x, t) .
$$

Similar to the constrained Allen-Cahn dynamics, evolution induced by equation (4.29) conserves the total integral of the field $u(x)$ (albeit in a different, "local" fashion). This conservation appears because the role of the diffusivity matrix is taken on by the degenerate $-\partial_{x x}$ operator, which means that we are in the hard constraint scenario again. It is fairly straightforward to derive the reduced diffusivity matrix formally applying the recipe from Remark 2.5 to Theorem 2.2: parametrizing the multi-kink functions via $\zeta_{\alpha}$, defined so that the kink locations may be expressed as $z_{\alpha}=\left(\zeta_{\alpha+1}+\right.$ 
$\left.\zeta_{\alpha}\right) / 2$ for $\alpha<N$ and $z_{N}=\left(\zeta_{1}+\zeta_{N}+C\right) / 2$ (with a suitably chosen constant $C$ ), we obtain

$$
D_{\alpha \beta}(\zeta) \approx \frac{\delta_{\alpha \beta}}{\ell_{\alpha}}-\frac{(-1)^{\alpha+\beta}\left(\ell_{\alpha}+\ell_{\beta}\right)}{N \ell_{\alpha} \ell_{\beta}} .
$$

Here $\ell_{\alpha}=\left(\zeta_{\alpha+1}-\zeta_{\alpha-1}\right) / 2=z_{\alpha}-z_{\alpha-1}$ are the domain lengths. This implies that without the contribution from the the reduced energy the process $\zeta_{t}$ obeys

$$
\dot{\boldsymbol{\zeta}}_{t}=\sqrt{2 \tau} D\left(\boldsymbol{\zeta}_{t}\right) \int_{0}^{L} \partial_{x}^{-1} \frac{\partial v\left(x \mid \boldsymbol{\zeta}_{\boldsymbol{t}}\right)}{\partial \boldsymbol{\zeta}} \circ \xi(x, t) \mathrm{d} x .
$$

Equations for the domain lengths $\ell_{\alpha}$ may be recovered immediately since the righthand side of (4.31) only depends in $\ell_{t}$ and $\ell_{\alpha}=\left(\zeta_{\alpha+1}-\zeta_{\alpha-1}\right) / 2$. However, one has to understand whether the effective energy, $h(\boldsymbol{z})$ (logarithm of the "product of nonzero eigenvalues" of the Hessian of the energy), contributes into the kink dynamics. This issue requires more insight into the associated spectral problem involving a fourth order differential operator and will be considered elsewehere.

4.4. Generalization to several spatial dimensions. The formalism developed above can in principle be extended to Allen-Cahn type equations in which the spatial variable $x$ is two (or higher) dimensional, e.g., to the following stochastic PDE:

$$
u_{t}(x, t)=\Delta u+\frac{1}{\epsilon^{2}}\left(u-u^{3}\right)+\sqrt{2 \tau} \xi(x, t) .
$$

It is known that in the limit of small $\epsilon$ the deterministic evolution reduces to the motion by mean curvature of the interfaces between the stable \pm 1 phases; see, e.g., $[15,24]$ for matched asymptotics, or $[7,10]$ for rigorous analysis. Indeed, a formal derivation is possible which leads to stochastically-perturbed motion by mean curvature [13]; however a rigorous justification of this derivation is lacking. The main issues are that the reduced dynamics itself is infinite-dimensional (evolution of curves or surfaces) and that rigorously interpreting equation (4.32) for $x \in \mathbb{R}^{d}, d>1$ is not straightforward.

\section{Proofs and discussion}

Here we provide the proof of Theorem 2.2 and other propositions from the previous sections. In particular, in section 5.2 we discuss convergence of invariant measures and in section 5.3 we discuss the hard constraint scenario of degenerate diffusivity.

5.1. Proof of Theorem 2.2. We now proceed to prove Theorem 2.2. We begin with an immediate simplification of equation (2.1), which is obtained by observing that its general form is equivalent to

$$
\mathrm{d} \boldsymbol{X}_{t}=-\nabla E\left(\boldsymbol{X}_{t}\right)+\sqrt{2 \tau} \sigma \mathrm{d} \boldsymbol{W}_{t}, \quad \sigma \sigma^{T}=I .
$$

This is because (2.1) may be transformed into (5.1) by the coordinate change $\boldsymbol{x}=J^{T} \boldsymbol{x}^{\prime}$, where $J^{T} J=D$, which is always possible since the matrix $D$ is symmetric and positivedefinite.

We proceed in three steps. First we state the theorem due to Katzenberger [18], which is the starting point of our computations. Next, we explicitly compute all the ingredients that are necessary for its application in our setting. And finally, we derive the formulas that appear in the statement of Theorem 2.2. 
5.1.1. Katzenberger's Theorem. We first state an abstract result which is the main tool used in our proof. Note that we state this result in an altered form most suitable for the use in our study, and with more demanding assumptions (e. g., in various places where we require differentiability, Lipschitz continuity is sufficient). We consider a diffusion process on $\mathbb{R}^{n}$ driven by a $d$-dimensional Brownian motion $\boldsymbol{W}_{t}$,

$$
\mathrm{d} \boldsymbol{X}_{t}^{\epsilon}=\boldsymbol{b}\left(\boldsymbol{X}_{t}^{\epsilon}\right) \mathrm{d} t-\frac{1}{\epsilon} \nabla V\left(\boldsymbol{X}_{t}^{\epsilon}\right) \mathrm{d} t+\sqrt{2 \tau} \sigma \mathrm{d} \boldsymbol{W}_{t},
$$

assuming that $\sigma \sigma^{T}=I$, and $V(\boldsymbol{x})$ and $\mathcal{M}$ are as described in Assumption 2.1.

Theorem 5.1. (Katzenberger) Let $\boldsymbol{b}(\boldsymbol{x}) \in C^{1}$. Consider a family of initial conditions $\boldsymbol{X}_{0}^{\epsilon}$ converging to some $\boldsymbol{X}_{0} \in \mathcal{M}$ as $\epsilon \rightarrow 0$, and let $\mathcal{U}$ be a compact neighborhood of $\boldsymbol{X}_{0}$ on $\mathcal{M}$. Then for all $\delta>0$,

$$
\lim _{\epsilon \rightarrow 0} \mathbb{P}\left(\sup _{0 \leq t \leq T}\left|\boldsymbol{X}_{t}^{\epsilon}-\boldsymbol{X}_{t}\right|>\delta\right)=0
$$

where $T=\inf \left\{t \geq 0 \mid \boldsymbol{X}_{t} \notin \mathcal{U}\right\}$ and $\boldsymbol{X}_{t}$ is a process that obeys the Itô $S D E$

$$
\mathrm{d} \boldsymbol{X}_{t}=\left[\nabla \boldsymbol{\phi}\left(\boldsymbol{X}_{t}\right) \boldsymbol{b}\left(\boldsymbol{X}_{t}\right)+\tau \Delta \phi\left(\boldsymbol{X}_{t}\right)\right] \mathrm{d} t+\sqrt{2 \tau} \nabla \boldsymbol{\phi}\left(\boldsymbol{X}_{t}\right) \sigma \mathrm{d} \boldsymbol{W}_{t} .
$$

Here $\boldsymbol{\phi}(\boldsymbol{x})$ is the limiting map of the gradient flow generated by $V(\boldsymbol{x})$, i.e., $\boldsymbol{\phi}$ maps an arbitrary point $\boldsymbol{x}$ to the $t=\infty$ limit of the flow $\varphi(\cdot, t)$ satisfying the following ODE ( $\boldsymbol{x}$ plays the role of a parameter)

$$
\frac{\mathrm{d}}{\mathrm{d} t} \boldsymbol{\varphi}(\boldsymbol{x}, t)=-\nabla V(\boldsymbol{\varphi}(\boldsymbol{x}, t)), \quad \boldsymbol{\varphi}(\boldsymbol{x}, 0)=\boldsymbol{x} .
$$

Throughout the rest of this discussion, for notational brevity, we will use the Einstein summation convention of summing over repeated indices. Moreover, we will denote the partial derivatives with respect to $x_{i}$ by $\partial_{i}$.

In the proof of Theorem 2.2, we will need equation (5.4) to be recast into the Stratonovich form, which we find as follows. Using the fact that $\nabla \phi(\boldsymbol{x})$ is an orthogonal projector (and thus symmetric and idempotent, see the following section), differentiating the identity $\phi(\phi(x))=\phi(x)$ twice to obtain $\partial_{j j}^{2} \phi^{i}(\boldsymbol{x})=\partial_{k} \phi^{l}(\boldsymbol{x}) \partial_{k l}^{2} \phi^{i}(\boldsymbol{x})+$ $\partial_{j} \phi^{i}(\boldsymbol{x}) \partial_{k k}^{2} \phi^{j}(\boldsymbol{x})$, and observing that the first term on the right-hand side is exactly the Itô correction, we find the Stratonovich SDE corresponding to (5.4) to be

$$
\mathrm{d} \boldsymbol{X}_{t}=\nabla \boldsymbol{\phi}\left(\boldsymbol{X}_{t}\right)\left[\left(\boldsymbol{b}\left(\boldsymbol{X}_{t}\right)+\tau \Delta \boldsymbol{\phi}\left(\boldsymbol{X}_{t}\right)\right) \mathrm{d} t+\sqrt{2 \tau} \sigma \circ \mathrm{d} \boldsymbol{W}_{t}\right] .
$$

Our goal is to derive equations for the reduced process, $\boldsymbol{X}_{t}$, in the intrinsic coordinates on $\mathcal{M}$. In order to do this, let us assume that $\mathcal{M}$ is parameterized by $\boldsymbol{z} \in \mathbb{R}^{m}$ via a map $\boldsymbol{x}=\boldsymbol{\psi}(\boldsymbol{z})$. We now have to present a process, $\boldsymbol{Z}_{t}$, such that $\boldsymbol{X}_{t}=\boldsymbol{\psi}\left(\boldsymbol{Z}_{t}\right)$ satisfies (5.4). Note that, since changes of variables are easier to carry out in the Stratonovich form, we will from here on use (5.6) rather than (5.4). We will begin the proof by explicitly computing the relevant derivatives of the fast flow map, $\phi(x)$. 
5.1.2. Derivatives of the fast flow map. The first step in the proof of Theorem 2.2 is the computation of the gradients $\partial_{j} \phi^{i}(\boldsymbol{x})$ for $\boldsymbol{x} \in \mathcal{M}$. Taking into account the results of [11] on the smoothness of $t=\infty$ maps of dynamical systems, we find from (5.5) that

$$
\partial_{j} \phi^{i}(\boldsymbol{x})=\lim _{t \rightarrow \infty} \partial_{j} \varphi^{i}(\boldsymbol{x}, t)
$$

where

$$
\frac{\mathrm{d}}{\mathrm{d} t} \partial_{j} \varphi^{i}(\boldsymbol{x}, t)=-\partial_{i k}^{2} V(\boldsymbol{x}) \partial_{j} \varphi^{k}(\boldsymbol{x}, t), \quad \partial_{j} \varphi^{i}(\boldsymbol{x}, 0)=\delta_{i j} .
$$

Since $\boldsymbol{x} \in \mathcal{M}$ and $\mathcal{M}$ is a manifold of minimizers for the potential $V(\boldsymbol{x}), \boldsymbol{x}$ is a fixed point of equation (5.5). Therefore, (5.8) is a linear system with constant coefficients and

$$
\partial_{j} \varphi^{i}(\boldsymbol{x}, t)=[\exp \{-H(\boldsymbol{x}) t\}]_{i j}, \quad H_{i j}(\boldsymbol{x})=\partial_{i j}^{2} V(\boldsymbol{x}) .
$$

Observe that the Hessian, $H(\boldsymbol{x})$, is symmetric and positive semi-definite; its kernel is the tangent space of $\mathcal{M}$ at $\boldsymbol{x}$, while its range is the orthogonal complement.

We now change the coordinates to a frame in which the Hessian, $H(\boldsymbol{x})$, is diagonal, which can always be achieved by a linear change of variables. Let us denote the eigenvalues of $H(\boldsymbol{x})$ by $\lambda_{i}, i=0 \ldots n$. In such a frame we have

$$
\partial_{j} \varphi^{i}(\boldsymbol{x}, t)=\operatorname{diag}\left\{\mathrm{e}^{-\lambda_{i}(\boldsymbol{x}) t}\right\} .
$$

Taking the limit $t \rightarrow \infty$, we immediately see that $\partial_{j} \phi^{i}(\boldsymbol{x})$ is the orthogonal projector on the tangent space of $\mathcal{M}$ at $\boldsymbol{x}$. This implies that if $\mathcal{M}$ is parameterized by a function $\boldsymbol{x}=\boldsymbol{\psi}(\boldsymbol{z}) \in \mathcal{M}, \partial_{j} \phi^{i}(\boldsymbol{x})$ is given by

$$
\partial_{j} \phi^{i}(\boldsymbol{x})=\partial_{\alpha} \psi^{i}(\boldsymbol{z}) G_{\alpha \beta}^{-1}(\boldsymbol{z}) \partial_{\beta} \psi^{j}(\boldsymbol{z}), \text { where } \quad G_{\alpha \beta}(\boldsymbol{z})=\partial_{\alpha} \psi^{i}(\boldsymbol{z}) \partial_{\beta} \psi^{i}(\boldsymbol{z}) .
$$

Here $\partial$ with Greek subscripts denotes differentiation with respect to the $\boldsymbol{z}$-variables. Let us now compute the second derivatives, $\partial_{j k}^{2} \phi^{i}(\boldsymbol{x})$. We have

$$
\partial_{j k}^{2} \phi^{i}(\boldsymbol{x})=\lim _{t \rightarrow \infty} \partial_{j k}^{2} \varphi^{i}(\boldsymbol{x}, t)
$$

where

$$
\frac{\mathrm{d}}{\mathrm{d} t} \partial_{j k}^{2} \varphi^{i}(\boldsymbol{x}, t)=-\partial_{i l}^{2} V(\boldsymbol{x}) \partial_{j k}^{2} \varphi^{l}(\boldsymbol{x}, t)-\partial_{i l l^{\prime}}^{3} V(\boldsymbol{x}) \partial_{j} \varphi^{l}(\boldsymbol{x}, t) \partial_{k} \varphi^{l^{\prime}}(\boldsymbol{x}, t)
$$

with the initial condition $\partial_{j k}^{2} \varphi^{i}(\boldsymbol{x}, 0)=0$. This is a linear nonhomogeneous equation with constant coefficients solved by (taking (5.9) into account)

$$
\partial_{j k}^{2} \varphi^{i}(\boldsymbol{x}, t)=-\partial_{k^{\prime} l l^{\prime}}^{3} V(\boldsymbol{x}) \int_{0}^{t} \partial_{k^{\prime}} \varphi^{i}(\boldsymbol{x}, t-s) \partial_{j} \varphi^{l}(\boldsymbol{x}, s) \partial_{k} \varphi^{l^{\prime}}(\boldsymbol{x}, s) \mathrm{d} s .
$$

Again, in the coordinate frame that diagonalizes the Hessian we obtain the more explicit expression

$$
\partial_{j k}^{2} \varphi^{i}(\boldsymbol{x}, t)=-\mathrm{e}^{-\lambda_{i}(\boldsymbol{x}) t} \partial_{i j k}^{3} V(\boldsymbol{x}) \int_{0}^{t} \mathrm{e}^{\left(\lambda_{i}(\boldsymbol{x})-\lambda_{j}(\boldsymbol{x})-\lambda_{k}(\boldsymbol{x})\right) s} \mathrm{~d} s .
$$


We now observe from equation (5.15) that if $\lambda_{i}(\boldsymbol{x}) \neq 0$, then $\partial_{j k}^{2} \varphi^{i}(\boldsymbol{x}, t) \rightarrow 0$ as $t \rightarrow \infty$. This is because if $\lambda_{j}(\boldsymbol{x})+\lambda_{k}(\boldsymbol{x})>0$, the exponent in front of the integral on the righthand side of (5.15) dominates and if $\lambda_{j}(\boldsymbol{x})=\lambda_{k}(\boldsymbol{x})=0$, then $\partial_{i j k}^{3} V(\boldsymbol{x})=0$ (since $\mathcal{M}$ is the manifold of minimizers of $V(\boldsymbol{x})$, the latter has to be differentiated twice in the normal direction to obtain a nonzero result). If $\lambda_{i}(\boldsymbol{x})=0$, then $\partial_{i j k}^{3} V(\boldsymbol{x}) \neq 0$ only if $\lambda_{j}(\boldsymbol{x})=\lambda_{k}(\boldsymbol{x}) \neq 0$; for such indices we find

$$
\partial_{j k}^{2} \varphi^{i}(\boldsymbol{x}, t)=-\partial_{i} \lambda_{j}(\boldsymbol{x}) \int_{0}^{t} \mathrm{e}^{-2 \lambda_{j}(\boldsymbol{x}) s} \mathrm{~d} s \longrightarrow-\frac{1}{2} \partial_{i} \ln \lambda_{j}(\boldsymbol{x}) \quad \text { as } \quad t \rightarrow \infty
$$

(no summation over repeated indices in this formula). In particular, denoting the product of the nonzero eigenvalues of the Hessian of $V(\boldsymbol{\psi}(\boldsymbol{z}))$ by $h(\boldsymbol{z})$, we may represent the term $\nabla \phi(\boldsymbol{x}) \Delta \phi(\boldsymbol{x})$ in (5.4) as

$$
\partial_{j} \phi^{i}(\boldsymbol{x}) \partial_{k k}^{2} \phi^{j}(\boldsymbol{x})=-\frac{1}{2} \partial_{j} \phi^{i}(\boldsymbol{x}) \partial_{j} \ln h(\boldsymbol{z}) .
$$

Note that even though we derived (5.11) and (5.17) in particular coordinate frames, the final expressions are invariant with respect to the coordinate choice.

5.1.3. Derivation of the final formula. Once we have all the ingredients in (5.6) explicitly computed, we may represent it as

$$
\mathrm{d} X_{t}^{i}=\partial_{\alpha} \psi^{i}\left(\boldsymbol{Z}_{t}\right) G_{\alpha \beta}^{-1}\left(\boldsymbol{Z}_{t}\right) \partial_{\beta} \psi^{j}\left(\boldsymbol{Z}_{t}\right)\left[-\partial_{j} F\left(\boldsymbol{Z}_{t}\right) \mathrm{d} t+\sqrt{2 \tau} \sigma_{r}^{j} \circ \mathrm{d} W_{t}^{r}\right],
$$

where $F(\boldsymbol{z})$ is the reduced energy given by

$$
F(\boldsymbol{z})=U(\boldsymbol{\psi}(\boldsymbol{z}))+\frac{\tau}{2} \ln h(\boldsymbol{z}) .
$$

Since for Stratonovich equations we have $\mathrm{d} X_{t}^{i}=\partial_{\alpha} \psi^{i}\left(\boldsymbol{Z}_{t}\right) \mathrm{d} Z_{t}^{\alpha}$, it becomes immediate that $\boldsymbol{X}_{t}=\boldsymbol{\psi}\left(\boldsymbol{Z}_{t}\right)$ will satisfy equation (5.18), provided $\boldsymbol{Z}_{t}$ satisfies

$$
\mathrm{d} Z_{t}^{\alpha}=-G_{\alpha \beta}^{-1}\left(\boldsymbol{Z}_{t}\right) \partial_{\beta} F\left(\boldsymbol{Z}_{t}\right) \mathrm{d} t+\sqrt{2 \tau} \hat{\sigma}_{r}^{\alpha}\left(\boldsymbol{Z}_{t}\right) \circ \mathrm{d} W_{t}^{r},
$$

where the matrix $\hat{\sigma}_{r}^{\alpha}(\boldsymbol{z})$ is given by

$$
\hat{\sigma}_{r}^{\alpha}(\boldsymbol{z})=G_{\alpha \beta}^{-1}(\boldsymbol{z}) \partial_{\beta} \psi^{j}(\boldsymbol{z}) \sigma_{r}^{j} .
$$

Finally, we obtain formulas (2.5) and (2.6) by changing the $\boldsymbol{x}$ variables to $\boldsymbol{x}=J^{T} \boldsymbol{x}^{\prime}$, where $J^{T} J=D$ and dropping the primes in final formulas. This concludes the proof of Theorem 2.2.

5.2. Convergence of the invariant measure. Let us recall that the original equation (2.1) possesses the invariant measure (2.7), which is of the form $\mathrm{d} \mu^{\epsilon}(\boldsymbol{x})=$ $\rho^{\epsilon}(\boldsymbol{x}) \mathrm{d} \boldsymbol{x}$, where

$$
\rho^{\epsilon}(\boldsymbol{x})=\frac{1}{\mathcal{Z}^{\epsilon}} \mathrm{e}^{-E^{\epsilon}(\boldsymbol{x}) / \tau}, \quad \mathcal{Z}^{\epsilon}=\int_{\mathbb{R}^{n}} \mathrm{e}^{-E^{\epsilon}(\boldsymbol{x}) / \tau} \mathrm{d} \boldsymbol{x}, \quad E^{\epsilon}(\boldsymbol{x})=U(\boldsymbol{x})+\frac{1}{\epsilon} V(\boldsymbol{x}) .
$$

(Here we assume that the normalizing constant $\mathcal{Z}^{\epsilon}$ exists, i.e., the invariant measure is finite. This, however, does not need to be the case for the calculation below and merely affects the scaling constant.) The following proposition describes what happens to the measure $\rho^{\epsilon}(\boldsymbol{x})$ as $\epsilon \rightarrow 0$. 
Proposition 5.2. (Concentration of the invariant measure) Let $V(\boldsymbol{x})$ and $\mathcal{M}$ be as described in Assumption 2.1 and $\rho^{\epsilon}(\boldsymbol{x})$ exist. Then, in the limit as $\epsilon \rightarrow 0, \mathrm{~d} \mu^{\epsilon}(\boldsymbol{x})$ converges to a measure on $\mathcal{M}$, whose density with respect to the Hausdorff measure is given by

$$
\rho(\boldsymbol{x})=Z^{-1} \exp \{-F(\boldsymbol{x}) / \tau\}, \quad F(\boldsymbol{x})=U(\boldsymbol{x})+\frac{\tau}{2} \ln h(\boldsymbol{x}),
$$

where $h(\boldsymbol{x})$ is the product of nonzero eigenvalues of the Hessian of $V(\boldsymbol{x})$ and $\mathcal{Z}$ is the normalizing factor. That is, for any test-function $\varphi(\boldsymbol{x})$ the following is true:

$$
\lim _{\epsilon \rightarrow 0} \int_{\mathbb{R}^{n}} \varphi(\boldsymbol{x}) \rho^{\epsilon}(\boldsymbol{x}) \mathrm{d} \boldsymbol{x}=\int_{\mathcal{M}} \varphi(\boldsymbol{x}) \rho(\boldsymbol{x}) \mathrm{d} s(\boldsymbol{x}) .
$$

Here $\mathrm{d} s(\boldsymbol{x})$ is the volume element on $\mathcal{M}$ inherited from $\mathrm{d} \boldsymbol{x}$ of $\mathbb{R}^{n}$ (the Hausdorff measure).

SkETCH OF THE PROOF. By assumption, the fast potential $V(\boldsymbol{x})$ attains its minima on the manifold $\mathcal{M}$. This implies that in the limit as $\epsilon \rightarrow 0$ the invariant measure becomes concentrated on $\mathcal{M}$ and we may compute it using the saddle-point method [21]. For any smooth function $\varphi(\boldsymbol{x})$ we have

$$
\left\langle\rho^{\epsilon}, \varphi\right\rangle=\int \rho^{\epsilon}(\boldsymbol{x}) \varphi(\boldsymbol{x}) \mathrm{d} \boldsymbol{x}=\frac{1}{\mathcal{Z}^{\epsilon}} \int \exp \left\{-\frac{1}{\epsilon \tau} V(\boldsymbol{x})\right\} \mathrm{e}^{-U(\boldsymbol{x}) / \tau} f(\boldsymbol{x}) \mathrm{d} \boldsymbol{x} .
$$

The principal contribution to the integral in (5.25) comes from an $\mathcal{O}(\epsilon)$ neighborhood of $\mathcal{M}$, in which we may decompose (5.25) into an integral over $\mathcal{M}$ and an integral over the directions normal to $\mathcal{M}$. This amounts to making a change of variables,

$$
\boldsymbol{x}=\boldsymbol{\Psi}(\boldsymbol{y}, \boldsymbol{z}),
$$

such that $\boldsymbol{\Psi}(0, \boldsymbol{z})$ parametrizes $\mathcal{M}$, while $\partial \boldsymbol{\Psi}(0, \boldsymbol{z}) / \partial \boldsymbol{z}$ and $\partial \boldsymbol{\Psi}(0, \boldsymbol{z}) / \partial \boldsymbol{y}$ are mutually orthogonal. In fact, we may require that the $(\boldsymbol{y}, \boldsymbol{z})$-coordinate frame be orthonormal in a sufficiently small neighborhood of $\mathcal{M}$. The (local) existence of such a variable change can be derived, e. g., from the Morse-Bott lemma [1].

For simplicity, we first consider only test functions $\varphi(\boldsymbol{x})$ whose support lies in one of the coordinate charts in which the local coordinate change (5.26) can be carried out. In this case, the integral (5.25) can be represented as

$$
\iint \exp \left\{-\frac{V(\boldsymbol{\Psi}(\boldsymbol{y}, \boldsymbol{z}))}{\epsilon \tau}\right\} \exp \left\{-\frac{U(\boldsymbol{\Psi}(\boldsymbol{y}, \boldsymbol{z}))}{\tau}\right\} \varphi(\boldsymbol{\Psi}(\boldsymbol{y}, \boldsymbol{z}))\left|\frac{\partial \boldsymbol{\Psi}(\boldsymbol{y}, \boldsymbol{z})}{\partial(\boldsymbol{y}, \boldsymbol{z})}\right| \mathrm{d} \boldsymbol{y} \mathrm{d} \boldsymbol{z} .
$$

Denoting $\boldsymbol{\psi}(\boldsymbol{z})=\boldsymbol{\Psi}(0, \boldsymbol{z})$ and evaluating the inner integral in equation (5.27) via the saddle-point method, we obtain

$$
\lim _{\epsilon \rightarrow 0}\left\langle\rho^{\epsilon}, \varphi\right\rangle=\frac{1}{\mathcal{Z}} \int\left|\frac{\partial^{2} V(\boldsymbol{\Psi}(0, \boldsymbol{z}))}{\partial y_{\mu} \partial y_{\nu}}\right|^{-1 / 2} \exp \left\{-\frac{U(\boldsymbol{\psi}(\boldsymbol{z}))}{\tau}\right\} \varphi(\boldsymbol{\psi}(\boldsymbol{z}))\left|\frac{\partial \boldsymbol{\Psi}(0, \boldsymbol{z})}{\partial(\boldsymbol{y}, \boldsymbol{z})}\right| \mathrm{d} \boldsymbol{z},
$$

where

$$
\mathcal{Z}=\lim _{\epsilon \rightarrow 0}(2 \pi \tau \epsilon)^{n-m} \mathrm{e}^{V(\mathcal{M}) / \epsilon \tau} \mathcal{Z}^{\epsilon}
$$


Since we have chosen the $(\boldsymbol{y}, \boldsymbol{z})$-frame to be orthonormal, the determinant of the $\boldsymbol{y}$-Hessian of $V(\boldsymbol{\Psi}(0, \boldsymbol{z}))$ is exactly the product of the nonzero eigenvalues of the $\boldsymbol{x}$ Hessian of $V(\boldsymbol{x})$ at $\boldsymbol{x}=\boldsymbol{\Psi}(0, \boldsymbol{z})$, while the Jacobian $|\partial \boldsymbol{\Psi}(0, \boldsymbol{z}) / \partial(\boldsymbol{y}, \boldsymbol{z})|$ is the scaling factor for the volume element on $\mathcal{M}$ in the $\boldsymbol{z}$-variables. Consequently, we find

$$
\lim _{\epsilon \rightarrow 0}\left\langle\rho^{\epsilon}, \varphi\right\rangle=\frac{1}{\mathcal{Z}} \int \frac{1}{\sqrt{h(\boldsymbol{z})}} \exp \left\{-\frac{U(\boldsymbol{\psi}(\boldsymbol{z}))}{\tau}\right\} \varphi(\boldsymbol{\psi}(\boldsymbol{z})) \mathrm{d} s(\boldsymbol{z}),
$$

where $\mathrm{d} s(\boldsymbol{z})$ is the volume element on $\mathcal{M}$ (the Hausdorff measure). Thus we recover (5.24), as claimed.

In general, the support of the test function $\varphi(\boldsymbol{x})$ cannot be covered by a single coordinate chart of the type described above. In this case one has to employ partitions of unity to compute the integral in (5.25) as a sum of integrals of the type (5.27) in local charts. We skip the details of this technical part of the proof.

5.3. Degenerate diffusivity and partial restriction. Here, we revisit the hard constraint scenario when the diffusivity matrix, $D$, in (2.1) is degenerate, which we briefly discussed in Remark 2.5. Recall that, in this case, the dynamics prescribed by (2.1) is constrained to the hyperplane Range $(D)+\boldsymbol{X}_{0} \subset \mathbb{R}^{n}$, and thus the limiting dynamics, in fact, occurs on $\tilde{\mathcal{M}}$, which is the intersection of $\mathcal{M}$ with this hyperplane. This time, rather than deriving reduced equations on $\tilde{\mathcal{M}}$, we still want to consider the reduced equation on $\mathcal{M}$. The reduced diffusivity, $\hat{D}(\boldsymbol{z})$, must remain degenerate to keep the constraint intact. Let us rename the matrices $D^{-1}$ and $\hat{D}^{-1}(\boldsymbol{z})$ in (2.6) into $G$ and $\hat{G}(\boldsymbol{z})$ respectively (since $D$ and $\hat{D}(\boldsymbol{z})$ are now singular and thus their normal inverses do not exist). Our goal is to derive formula (2.9) for a proper (providing the correct expression for $\hat{D}(\boldsymbol{z}))$ inversion of the matrix $\hat{G}(\boldsymbol{z})$.

Proposition 5.3. Assume that the submanifold $\tilde{\mathcal{M}}=\mathcal{M} \cap\left(\operatorname{Range}(D)+\boldsymbol{X}_{0}\right)$ is generic, i.e., $\tilde{\mathcal{M}}$ does not undergo topological changes with small variations of $\boldsymbol{X}_{0}$, and that Theorem 2.2 is applicable within each hyperplane Range $(D)+\boldsymbol{Y}_{0}$ for all $\boldsymbol{Y}_{0}$ in some neighborhood of $\boldsymbol{X}_{0}$. Then the conclusion of Theorem 2.2 and equation (2.5) for the reduced process, $\boldsymbol{Z}_{t}$, on $\mathcal{M}$ hold true provided the reduced diffusivity is given by

$$
\hat{D}(\boldsymbol{z})=P\left[P^{T} \hat{G} P+\tau Q^{T} Q\right]^{-1} P^{T}
$$

where $P(\boldsymbol{z})$ is an arbitrary projector on the tangent space of $\tilde{\mathcal{M}}$ at $\boldsymbol{z}, Q(\boldsymbol{z})=I-P(\boldsymbol{z})$, and $\tau>0$.

SKetch OF THE PROOF. Applying Theorem 2.2 we can obtain a reduced equation on $\tilde{\mathcal{M}}$ parametrized by some $\tilde{\boldsymbol{z}} \in \mathbb{R}^{\tilde{m}}: \mathrm{d} \tilde{\boldsymbol{Z}}_{t}=-\tilde{D}\left(\tilde{\boldsymbol{Z}}_{t}\right) \nabla \tilde{F}\left(\tilde{\boldsymbol{Z}}_{t}\right) \mathrm{d} t+\sqrt{2 \tau} \tilde{\sigma}\left(\tilde{\boldsymbol{Z}}_{t}\right) \circ \mathrm{d} \boldsymbol{W}_{t}$. Assuming that $\tilde{\mathcal{M}}$ may be parametrized (within the $\boldsymbol{z}$-space $\mathbb{R}^{m}$ ) via $\boldsymbol{z}=\boldsymbol{\zeta}(\tilde{\boldsymbol{z}}$ ) and using the Stratonovich change of variables formula for $\boldsymbol{Z}_{t}=\boldsymbol{\zeta}\left(\tilde{\boldsymbol{Z}}_{t}\right)$ we can then lift this equation to $\mathcal{M}$, obtaining an analogue of $(2.5)$ where the matrix $\hat{D}(\boldsymbol{z})$ is given by

$$
\hat{D}_{\alpha \beta}(\boldsymbol{z})=\sum_{\mu, \nu=1}^{\tilde{m}} \frac{\partial \zeta_{\alpha}(\tilde{\boldsymbol{z}})}{\partial \tilde{z}_{\mu}} \tilde{D}_{\mu \nu}(\tilde{\boldsymbol{z}}) \frac{\partial \zeta_{\beta}(\tilde{\boldsymbol{z}})}{\partial \tilde{z}_{\nu}}
$$

This formula, however, may well be too complicated to use, since it involves the matrix $\tilde{D}(\tilde{\boldsymbol{z}})$ and the gradients of $\boldsymbol{\zeta}(\tilde{\boldsymbol{z}})$, which are not necessarily easily available. Our goal is to show that $\hat{D}(\boldsymbol{z})$ may, in fact, be computed directly using the formula $(5.30)$. 
Let $G$ denote a symmetric matrix that inverts of $D$ from $(2.1)$ in its range, $H(G$ may be computed, e.g., using the result of Lemma 5.1 below). Consider the matrix $\hat{G}(\boldsymbol{z})$ given by

$$
\hat{G}_{\alpha \beta}(\boldsymbol{z})=\sum_{i, j=1}^{n} \frac{\partial \psi_{i}(\boldsymbol{z})}{\partial z_{\alpha}} G_{i j} \frac{\partial \psi_{j}(\boldsymbol{z})}{\partial z_{\beta}}
$$

Let $P(\tilde{\boldsymbol{z}})$ denote a particular projector on $H$, given by

$$
P_{\alpha \beta}(\tilde{\boldsymbol{z}})=\sum_{\mu, \nu=1}^{\tilde{m}} \frac{\partial \zeta_{\alpha}(\tilde{\boldsymbol{z}})}{\partial \tilde{z}_{\mu}} T_{\mu \nu}^{-1}(\tilde{\boldsymbol{z}}) \frac{\partial \zeta_{\beta}(\tilde{\boldsymbol{z}})}{\partial \tilde{z}_{\nu}}
$$

where

$$
T_{\mu \nu}(\tilde{\boldsymbol{z}})=\sum_{\alpha=1}^{m} \frac{\partial \zeta_{\alpha}(\tilde{\boldsymbol{z}})}{\partial \tilde{z}_{\mu}} \frac{\partial \zeta_{\alpha}(\tilde{\boldsymbol{z}})}{\partial \tilde{z}_{\nu}}
$$

We then have that $[\hat{D} \hat{G} P]_{\alpha \beta}(\boldsymbol{z})$ is given by

$$
\sum_{\eta, \xi, \mu, \nu=1}^{\tilde{m}} \sum_{\gamma, \delta=1}^{m} \sum_{i, j=1}^{n} \frac{\partial \zeta_{\alpha}(\tilde{\boldsymbol{z}})}{\partial \tilde{z}_{\mu}} \tilde{D}_{\mu \nu}(\tilde{\boldsymbol{z}}) \frac{\partial \zeta_{\delta}(\tilde{\boldsymbol{z}})}{\partial \tilde{z}_{\nu}} \frac{\partial \psi_{i}(\boldsymbol{z})}{\partial z_{\delta}} G_{i j} \frac{\partial \psi_{j}(\boldsymbol{z})}{\partial z_{\gamma}} \frac{\partial \zeta_{\gamma}(\tilde{\boldsymbol{z}})}{\partial \tilde{z}_{\eta}} T_{\eta \xi}^{-1}(\tilde{\boldsymbol{z}}) \frac{\partial \zeta_{\beta}(\tilde{\boldsymbol{z}})}{\partial \tilde{z}_{\xi}}
$$

Taking into account that (using the chain rule)

$$
\sum_{i, j=1}^{n} \sum_{\delta, \gamma=1}^{m} \frac{\partial \zeta_{\delta}(\tilde{\boldsymbol{z}})}{\partial \tilde{z}_{\nu}} \frac{\partial \psi_{i}(\boldsymbol{z})}{\partial z_{\delta}} G_{i j} \frac{\partial \psi_{j}(\boldsymbol{z})}{\partial z_{\gamma}} \frac{\partial \zeta_{\gamma}(\tilde{\boldsymbol{z}})}{\partial \tilde{z}_{\eta}}=\tilde{D}_{\nu \eta}^{-1}(\tilde{\boldsymbol{z}})
$$

we simplify $(5.35)$ to

$$
[\hat{D} \hat{G} P]_{\alpha \beta}(\boldsymbol{z})=\sum_{\mu, \nu, \eta, \xi=1}^{\tilde{m}} \frac{\partial \zeta_{\alpha}(\tilde{\boldsymbol{z}})}{\partial \tilde{z}_{\mu}} \tilde{D}_{\mu \nu}(\tilde{\boldsymbol{z}}) \tilde{D}_{\nu \eta}^{-1}(\tilde{\boldsymbol{z}}) T_{\eta \xi}^{-1}(\tilde{\boldsymbol{z}}) \frac{\partial \zeta_{\beta}(\tilde{\boldsymbol{z}})}{\partial \tilde{z}_{\xi}}=P_{\alpha \beta}(\tilde{\boldsymbol{z}})
$$

Summarizing all of the above, we see that $\hat{D}(\boldsymbol{z})$ is a symmetric matrix whose range is $H$ and which satisfies $\hat{D}(\boldsymbol{z}) \hat{G}(\boldsymbol{z}) P(\boldsymbol{z})=P(\boldsymbol{z})$, therefore by the Lemma 5.1 below, it is uniquely defined and may be computed by means of the formula (5.30), as claimed. $\square$

Lemma 5.1. Consider a subspace $H$ of $\mathbb{R}^{m}$. Let $G$ be a symmetric matrix which is positive on $H$, i.e., for all nonzero $\boldsymbol{x} \in H, \boldsymbol{x}^{T} G \boldsymbol{x}>0$. Then there exists a unique symmetric matrix $D$ whose range is precisely $H$ and which inverts $G$ in $H$, i.e., $D$ satisfies $D G P=P$, where $P$ is some projector on $H$. Moreover, $D$ may be represented as

$$
D=P\left[P^{T} G P+\tau Q^{T} Q\right]^{-1} P^{T},
$$

where $P$ is now an arbitrary projector on $H, Q=I-P$, and $\tau$ is an arbitrary positive constant.

Proof. First of all, let us show that the matrix in square brackets in (5.38) is invertible for all $\tau>0$. Choose an arbitrary $\boldsymbol{v} \in \mathbb{R}^{n}$ and represent it as $\boldsymbol{v}=\boldsymbol{x}+\boldsymbol{u}$ where $\boldsymbol{x} \in H, \boldsymbol{u} \in \operatorname{Range}(Q)$. We then have

$$
\boldsymbol{v}^{T}\left[P^{T} G P+\tau Q^{T} Q\right] \boldsymbol{v}=\boldsymbol{x}^{T} G \boldsymbol{x}+\tau \boldsymbol{u}^{T} \boldsymbol{u} \geq 0,
$$


with equality if and only if $\boldsymbol{x}=\boldsymbol{u}=0$. Therefore $P^{T} G P+\tau Q^{T} Q$ is positive (clearly, it is symmetric) and thus invertible.

To see that $D$ satisfies $D G P=P$, observe that

$$
\begin{aligned}
D G P & =P\left[P^{T} G P+\tau Q^{T} Q\right]^{-1} P^{T} G P \\
& =P\left[P^{T} G P+\tau Q^{T} Q\right]^{-1}\left(P^{T} G P+\tau Q^{T} Q\right) P=P .
\end{aligned}
$$

Now assume that some $D_{1}$ and $D_{2}$ satisfy the required conditions with some projectors $P_{1}$ and $P_{2}$, respectively. Then for all $\boldsymbol{x}, \boldsymbol{y} \in H$,

$$
\begin{aligned}
\boldsymbol{x}^{T} D_{1} G \boldsymbol{y}=\boldsymbol{x}^{T} D_{1} G P_{1} \boldsymbol{y} & =\boldsymbol{x}^{T} P_{1} \boldsymbol{y} \\
& =\boldsymbol{x}^{T} \boldsymbol{y} \\
& =\boldsymbol{x}^{T} P_{2} \boldsymbol{y}=\boldsymbol{x}^{T} D_{2} G P_{2} \boldsymbol{y}=\boldsymbol{x}^{T} D_{2} G \boldsymbol{y},
\end{aligned}
$$

i.e., $\boldsymbol{x}^{T}\left(D_{1}-D_{2}\right) G \boldsymbol{y}=0$. However, if $D_{1} \neq D_{2}$, there exists $\boldsymbol{x} \in H$ such that $\left(D_{1}-\right.$ $\left.D_{2}\right) \boldsymbol{x}=\boldsymbol{y} \neq 0$, but then $0=\boldsymbol{x}^{T}\left(D_{1}-D_{2}\right) G \boldsymbol{y}=\boldsymbol{y}^{T} G \boldsymbol{y}$ - a contradiction as $G$ is positive on $H$.

5.4. An estimate on the Ginzburg-Landau energy. Here we show that in the limit of small $\epsilon$, the deviations from the kink shape (4.6) are energetically expensive. For simplicity we will only consider the single-kink solutions. Consider the rescaled Ginzburg-Landau energy (rescaling is introduced in order to simplify the subsequent calculations),

$$
\mathcal{F}[u]=\int_{\mathbb{R}}\left[u_{x}^{2}+\left(1-u^{2}\right)^{2}\right] \mathrm{d} x,
$$

on functions satisfying $u(x) \rightarrow \pm 1$ as $x \rightarrow \pm \infty$. This energy is minimized by the family of single-kink solutions $v(x \mid Z)=\tanh (x-Z)$ with $\mathcal{F}[v]=8 / 3(2 \sqrt{2} / 3 \epsilon$ in the original scaling (4.3)). Without loss of generality let us set $Z=0$, and exclude the translations restricting perturbations of the kink shape to the subspace orthogonal to the translational mode, see below. Let $\mathcal{L}$ be (half) the second variation of the energy $\mathcal{F}[u]$ near the critical point $u(x)=\tanh x$,

$$
\mathcal{L}=\frac{1}{2} \mathrm{D}^{2} \mathcal{F}[\tanh x]=-\partial_{x x}^{2}+4-\frac{6}{\cosh ^{2} x} .
$$

The eigenvalues and eigenfunctions of $\mathcal{L}$ may be found explicitly:

$$
\begin{array}{ll}
\lambda_{0}=0, & \psi_{0}(x)=\cosh ^{-2} x \\
\lambda_{1}=3, & \psi_{1}(x)=\sinh x \cosh ^{-2} x .
\end{array}
$$

Here $\lambda_{0}$ corresponds to the translational mode (the eigenfunction $\psi_{0}(x)$ shifts the tanh-layer), whereas $\lambda_{1}$ corresponds to the shear mode $\left(\psi_{1}(x)\right.$ rips the tanh-layer in two). The following lemma is a variation of the Theorem 3.1 in [17].

Lemma 5.2. There exists $\nu>0$ such that whenever $\left(w, \psi_{0}\right)_{L^{2}}=0$ and $\|w\|_{H^{1}}<\nu$,

$$
\mathcal{F}[w(x)+\tanh x]-\frac{8}{3} \geq \frac{1}{3}\|w\|_{H^{1}}^{2} .
$$

Proof. By direct computation we can verify that

$$
\mathcal{F}[w(x)+\tanh x]-\frac{8}{3}=(w, \mathcal{L} w)_{L^{2}}+\int_{\mathbb{R}}[w(x)+4 \tanh x] w^{3}(x) \mathrm{d} x .
$$


From (5.44) we obtain that for any $w(x)$ satisfying $\left(w, \psi_{0}\right)_{L^{2}}=0$,

$$
(w, \mathcal{L} w)_{L^{2}} \geq 3\|w\|_{L^{2}}^{2} .
$$

From (5.43), using that $\left(w^{2}, \psi_{0}\right)_{L^{2}} \leq\|w\|_{L^{2}}^{2}$, we obtain that

$$
(w, \mathcal{L} w)_{L^{2}}=\left\|w_{x}\right\|_{L^{2}}^{2}+4\|w\|_{L^{2}}^{2}-6\left(w^{2}, \psi_{0}\right)_{L^{2}} \geq\left\|w_{x}\right\|_{L^{2}}^{2}-2\|w\|_{L^{2}}^{2} .
$$

Summing (5.47) and (5.48) we estimate the first term on the right-hand side of equation $(5.46)$ as

$$
(w, \mathcal{L} w)_{L^{2}} \geq \frac{1}{2}\|w\|_{H^{1}}^{2}
$$

In order to estimate the second term in (5.46), recollect that (by the Cauchy-Schwartz inequality)

$$
\|w\|_{\infty} \leq \frac{1}{\sqrt{2}}\|w\|_{H^{1}}
$$

and thus

$$
\begin{aligned}
& \left|\int_{\mathbb{R}}[w(x)+4 \tanh x] w^{3}(x) \mathrm{d} x\right| \leq\left[\frac{1}{\sqrt{2}}\|w\|_{H^{1}}+4\right]\|w\|_{L^{3}}^{3} \\
\leq & {\left[\frac{1}{\sqrt{2}}\|w\|_{H^{1}}+4\right]\|w\|_{\infty}\|w\|_{L^{2}}^{2} \leq 2 \sqrt{2}\|w\|_{H^{1}}^{3}+\frac{1}{2}\|w\|_{H^{1}}^{4} . }
\end{aligned}
$$

Finally, combining (5.51) with (5.49) and (5.46) we obtain that

$$
\mathcal{F}[w(x)+\tanh x]-\frac{8}{3} \geq \frac{1}{2}\|w\|_{H^{1}}^{2}-2 \sqrt{2}\|w\|_{H^{1}}^{3}-\frac{1}{2}\|w\|_{H^{1}}^{4} .
$$

Clearly, whenever $\|w\|_{H^{1}}$ is sufficiently small, $\mathcal{F}[w(x)+\tanh x]-8 / 3 \geq\|w\|_{H^{1}}^{2} / 3$, as claimed.

Corollary 5.4. Now let $u^{\epsilon}(x)=w(x / \epsilon \sqrt{2})+\tanh (x / \epsilon \sqrt{2})$. A straightforward rescaling shows that for the original Ginzburg-Landau energy (4.3) we have

$$
\mathcal{E}^{\epsilon}\left[u^{\epsilon}\right]-\mathcal{E}_{\mathrm{k}}^{\epsilon}=\frac{\sqrt{2}}{4 \epsilon}\left[\mathcal{F}[w(x)+\tanh x]-\frac{8}{3}\right] \geq \frac{\sqrt{2}}{12 \epsilon}\|w\|_{H^{1}}^{2} .
$$

Thus the deviations (in $H^{1}$ ) from the kink shape come with the $\mathcal{O}(1 / \epsilon)$ energy cost.

\section{REFERENCES}

[1] A. Banyaga and D. Hurtubise, Lectures on Morse Homology, Texts in the Mathematical Sciences, Springer, 2005.

[2] N. Berglund and B. Gentz, Geometric singular perturbation theory for stochastic differential equations, J. Differ. Eqs., 191, 1-54, 2003.

[3] D. Blömker, Stochastic Partial Differential Equataions and Surface Growth, Wissner-Verlag, 2000.

[4] D. Blömker, S. Maier-Paape and T. Wanner, Phase separation in stochastic Cahn-Hilliard models, A. Miranville (Ed.), Mathematical methods and models, Nova Science Publishers, 2005.

[5] L. Bronsard and R.V. Kohn, On the slowness of phase boundary motion in one space dimension, Commun. Pure Appl. Math., XLIII: 983-997, 1990. 
[6] J. Carr and R.L. Pego, Metastable patterns in solutions of $u_{t}=\epsilon^{2} u_{x x}-f(u)$, Commun. Pure Appl. Math., XLII: 523, 1989.

[7] X. Chen, Generation and propagation of the interface for reaction-diffusion equations, IMA Preprint Series, 637, 1990.

[8] W. E, W. Ren and E. Vanden-Eijnden, Minimum action method for the study of rare events, Commun. Pure Appl. Math., 52, 637-656, 2004.

[9] J.P. Eckmann and J. Rougemont, Coarsening by Ginzburg-Landau dynamics, Commun. Math. Phys., 199, 441, 1998.

[10] L.C. Evans, H.M. Soner and P.E. Souganidis, Phase transitions and generalized motion by mean curvature, Commun. Pure Appl. Math., XLV:1097, 1992.

[11] K.J. Falconer, Differentiation of the limit mapping in a dynamical system, Journal of London Mathematical Society, 2(27), 356-372, 1983.

[12] W.G. Faris and G. Jona-Lasinio, Large fluctuations for a nonlinear heat equation with noise, Journal of Physics A, 15, 3025-3055, 1982.

[13] I. Fatkullin, Coarsening in stochastically perturbed Ginzburg-Landau-type equations and statistical studies of Potts model, PhD Thesis, RPI, 2002.

[14] N. Fenichel, Geometric singular perturbation theory for ordinary differential equations, J. Differ. Eqs., 31, 53, 1979.

[15] P.C. Fife, Dynamics of internal layers and diffusive interfaces, SIAM, CBMS-NSF Regional Conference Series in Applied Mathematics, 53, 1988.

[16] M.I. Freidlin and A.D. Wentzell, Random perturbations of dynamical systems, Springer, 2nd edition, 1998.

[17] T. Funaki, The scaling limit for a stochastic PDE and the separation of phases, Probability Theory and Related Fields, 102, 221, 1995.

[18] G.S. Katzenberger, Solutions of a stochastic differential equation forced onto a manifold by a large drift, Annals of Probability, 19, 1587, 1991.

[19] R.V. Kohn, F. Otto, M.G. Reznikoff and E. Vanden-Eijnden, Action minimization and sharpinterface limits for the stochastic Allen-Cahn equation, Commun. Pure Appl. Math., 60, 393-438, 2007.

[20] R. Marcus, Monotone parabolic Itô equations, J. Functional Analysis, 6(6), 2402-2420, 1978.

[21] F.W.J. Olver, Asymptotics and Special Functions (AKP Classics), 2nd edition, AK Peters, 1997.

[22] E. Pardoux, Stochastic partial differential equations, a review, Bulletin des Sciences Mathématiques, 117, 29-47, 1993.

[23] L.C.G. Rogers and D. Williams, Diffusions, Markov Processes and Martingales: Volume 2, Itô Calculus, Cambridge University Press, 2nd edition, 2000.

[24] J. Rubinstein, P. Sternberg and J. Keller, Fast reaction, slow diffusion, and curve shortening, SIAM J. Appl. Math., 49(1), 116, 1989.

[25] T. Shardlow, Stochastic perturbations of the Allen-Cahn equation, Elec. J. Diff. Equ., 2000(47), $1-19,2000$. 